Supporting Information

\title{
A Convergent Formal Synthesis of Ecteinascidin 743
}

Junhao Jia, Ruijiao Chen, Yuanliang Jia, He Gu, Qin Zhou and Xiaochuan Chen*

Key Laboratory of Green Chemistry \& Technology of Ministry of Education, College of Chemistry, Sichuan University, Chengdu 610064, PR China

\section{E-mail: chenxc@scu.edu.cn}

Table of contents

A. Copies of ${ }^{1} \mathrm{H}$ and ${ }^{13} \mathrm{C}$ NMR spectra for compounds......................... 2 
Copies of ${ }^{1} \mathrm{H}$ and ${ }^{13} \mathrm{C}$ NMR spectra for compounds

${ }^{1} \mathrm{H}$ NMR spectrum of compound $17\left(400 \mathrm{MHz}, \mathrm{CD}_{3} \mathrm{OD}\right)$

岸<smiles>Cc1c(O)c(C[C@H](N)CO)cc2c1OCO2</smiles>

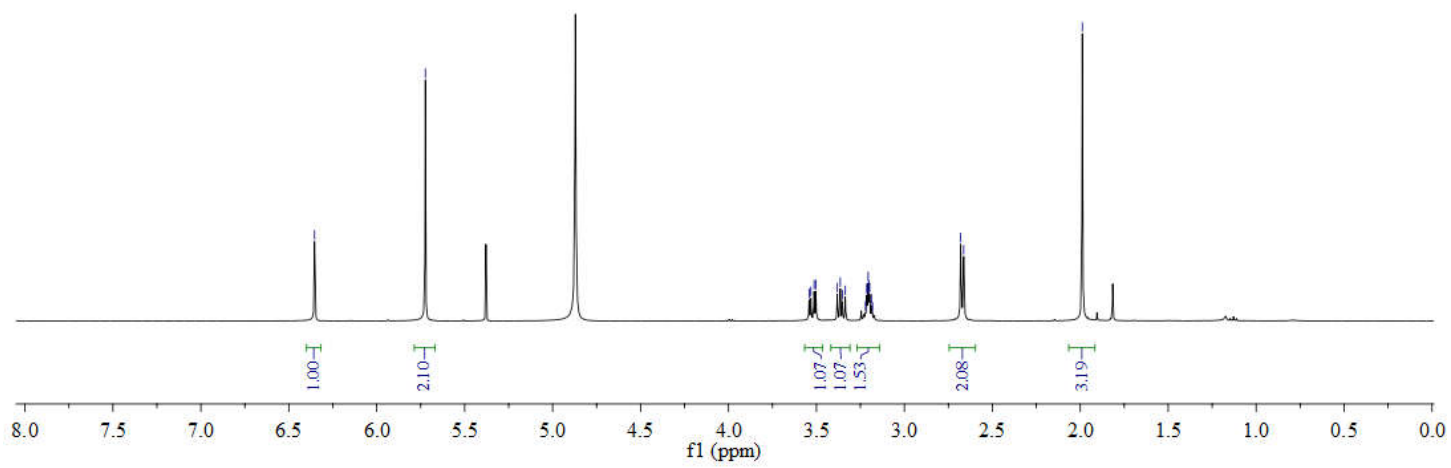

${ }^{13} \mathrm{C}$ NMR spectrum of compound 17 (100 MHz, CD $\left.{ }_{3} \mathrm{OD}\right)$<smiles>Cc1c(O)c(C[C@H](N)CO)cc2c1OCO2</smiles>

17
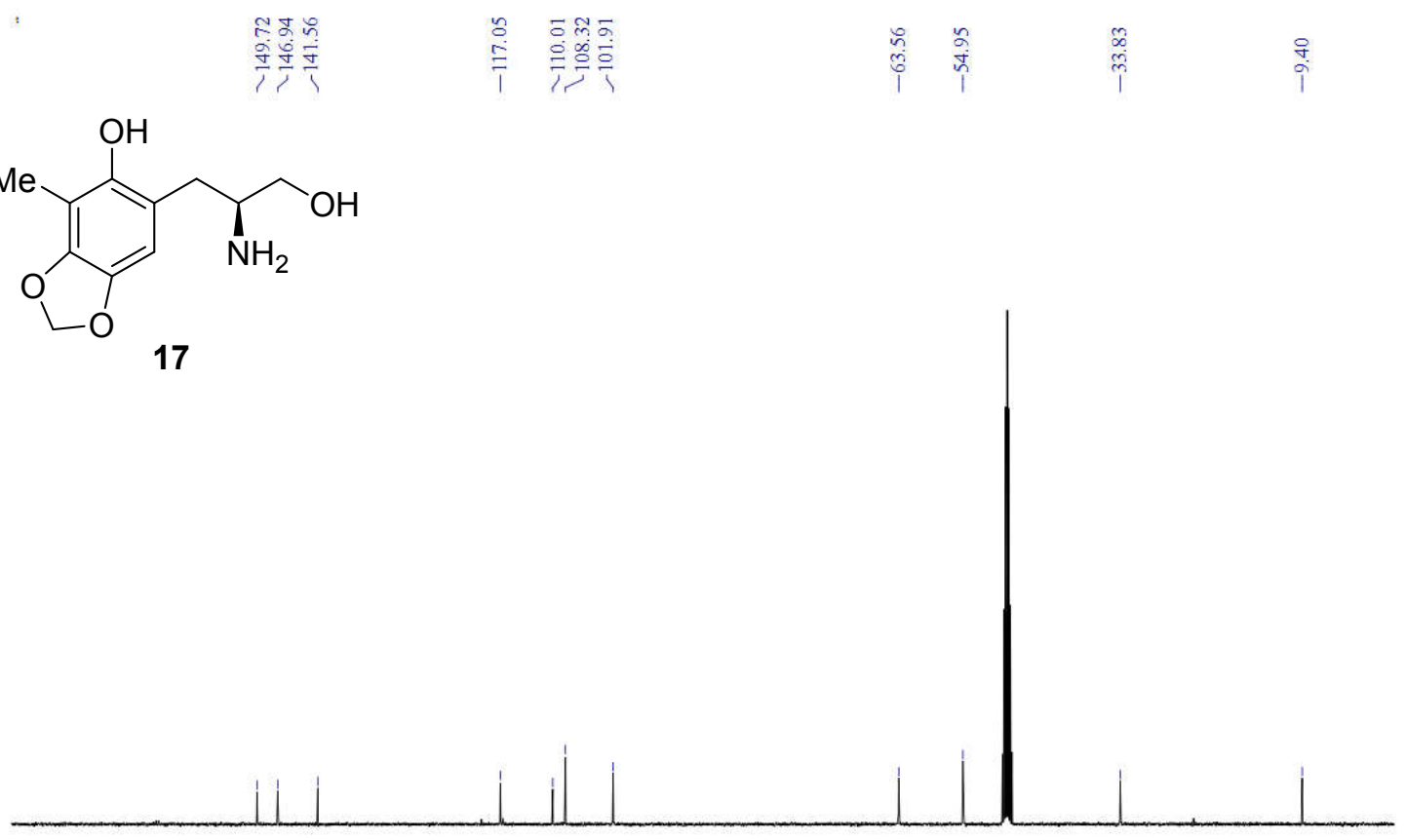

$\begin{array}{lllllllllllllllllll}180 & 170 & 160 & 150 & 140 & 130 & 120 & 110 & 100 & \begin{array}{c}90 \\ \mathrm{f} 1(\mathrm{ppm})\end{array} & 80 & 70 & 60 & 50 & 40 & 30 & 20 & 10 & 0\end{array}$ 
${ }^{1} \mathrm{H}$ NMR spectrum of compound $19\left(400 \mathrm{MHz}, \mathrm{CDCl}_{3}\right)$

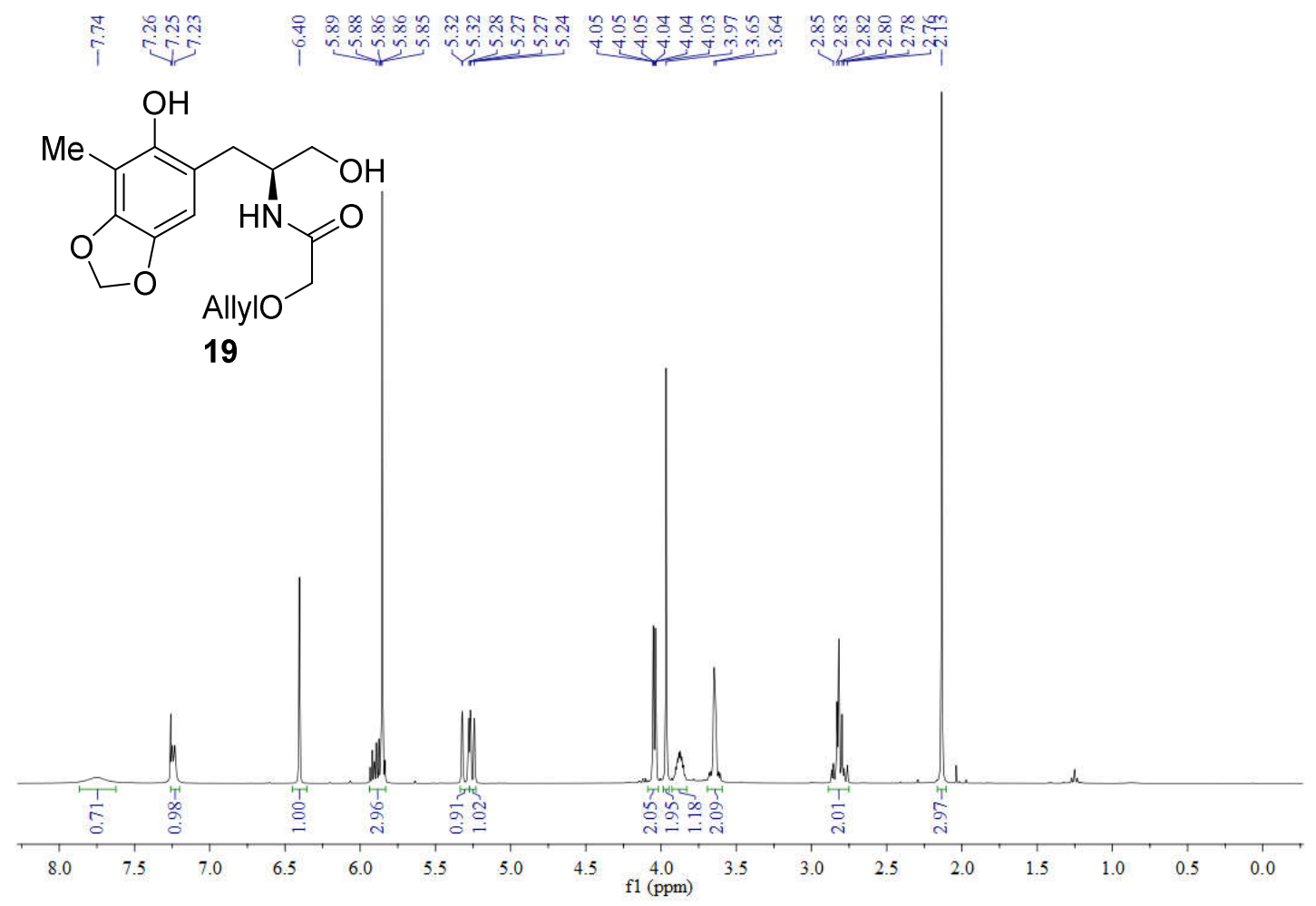

${ }^{13} \mathrm{C}$ NMR spectrum of compound $19\left(100 \mathrm{MHz}, \mathrm{CDCl}_{3}\right)$

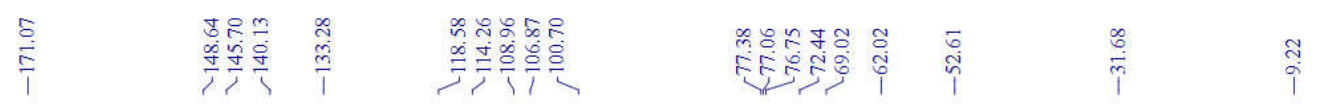<smiles>Cc1c(O)c(C[C@@H](CO)NC(=O)COCCOCCO)cc2c1OCO2</smiles>

19

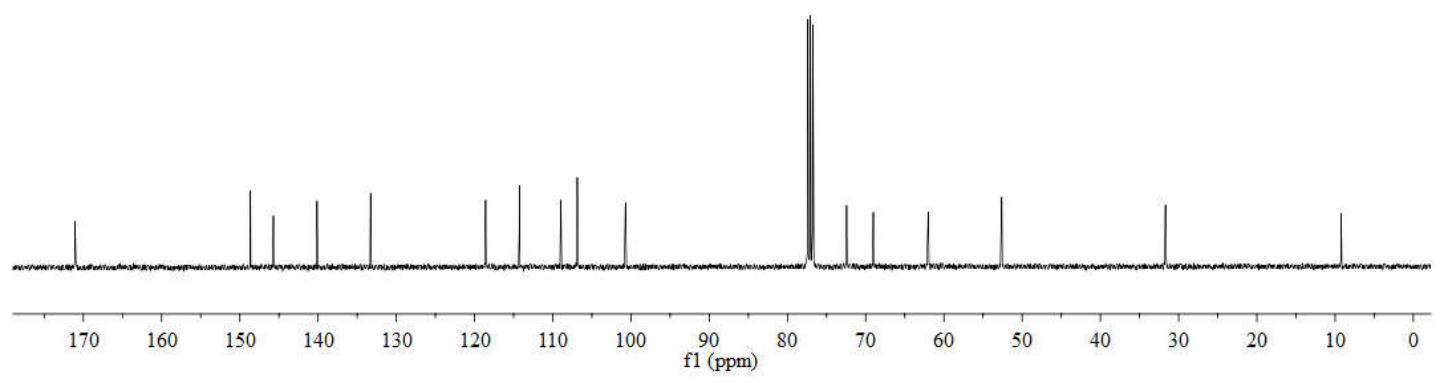


${ }^{1} \mathrm{H}$ NMR spectrum of compound $20\left(400 \mathrm{MHz}, \mathrm{CDCl}_{3}\right)$

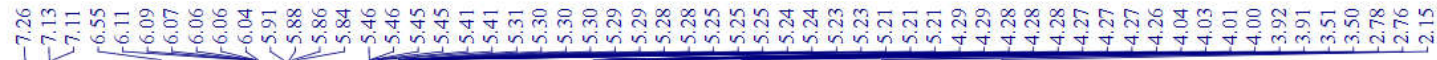<smiles>COc1c(CC(CO)NC(=O)CO[GaH2])cc2c(c1C)OCO2</smiles>

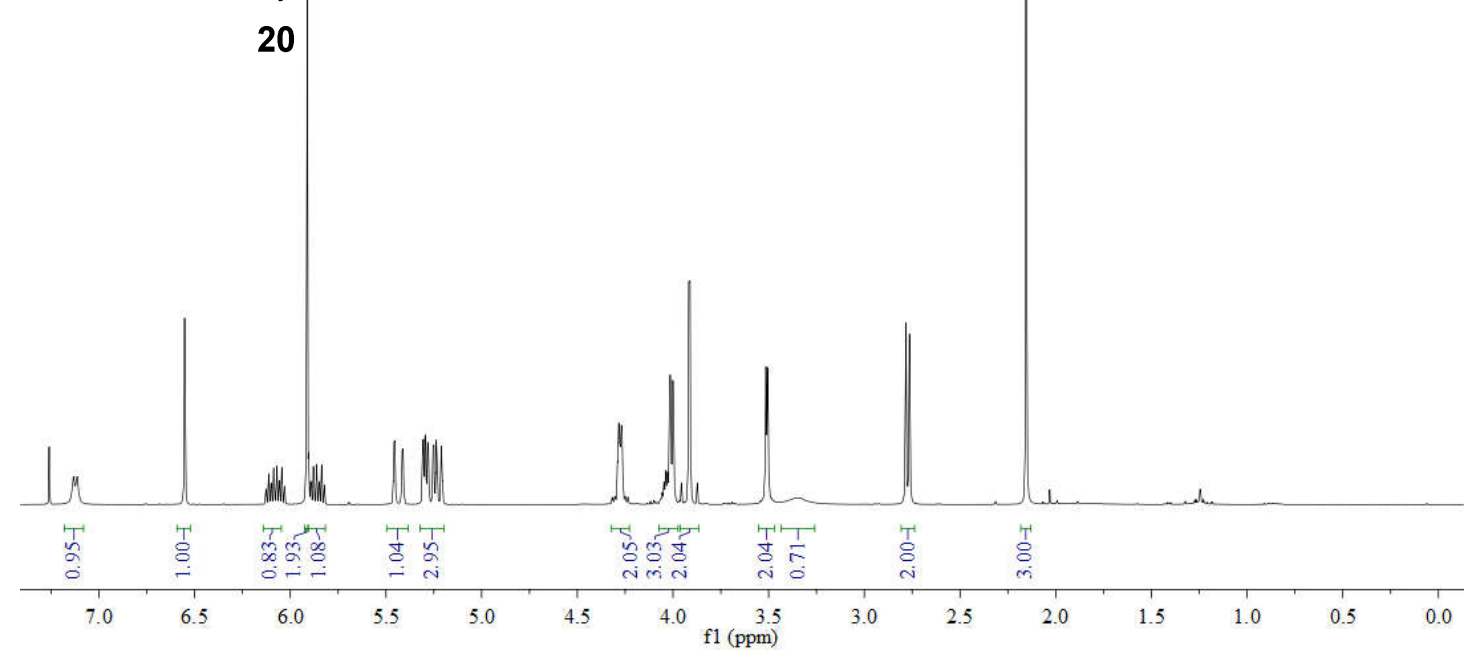

${ }^{13} \mathrm{C}$ NMR spectrum of compound $20\left(100 \mathrm{MHz}, \mathrm{CDCl}_{3}\right)$

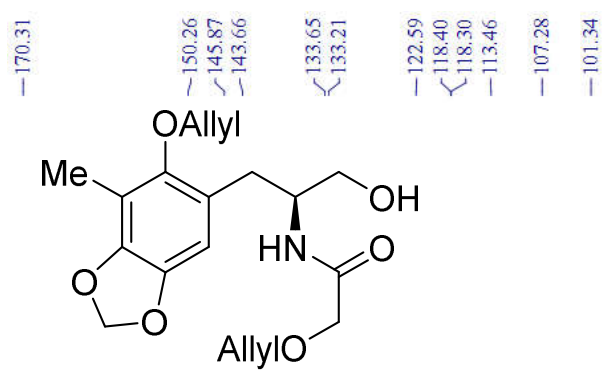

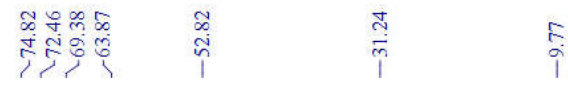

20

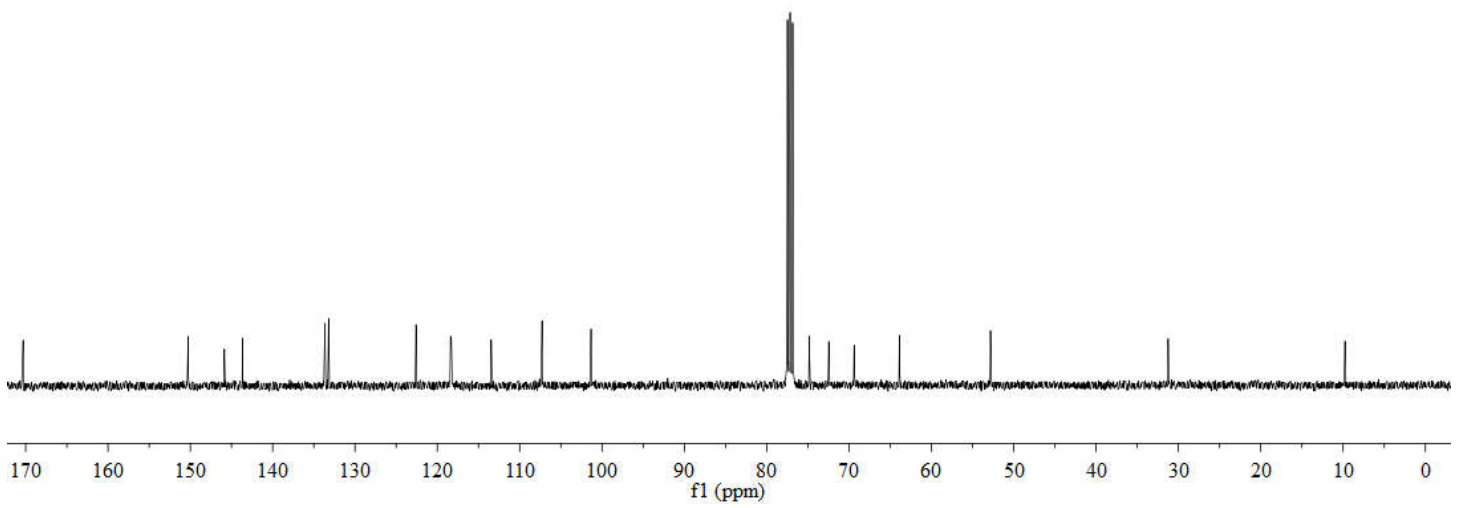


${ }^{1} \mathrm{H}$ NMR spectrum of compound $21\left(400 \mathrm{MHz}, \mathrm{CDCl}_{3}\right)$

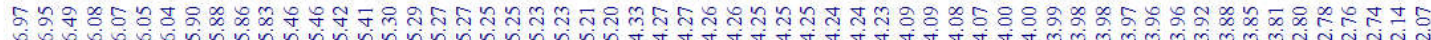<smiles>COc1c(C[C@@H](COC(C)=O)NC(=O)CO)cc2c(c1C)OCO2</smiles>

21

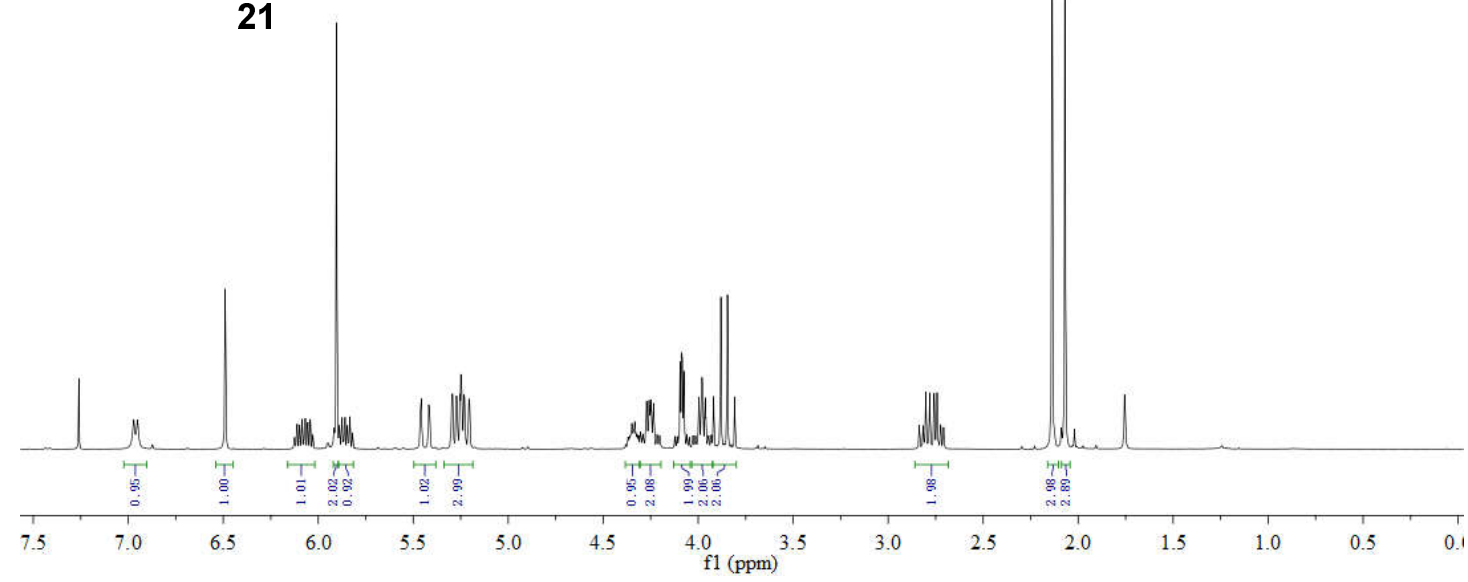

${ }^{13} \mathrm{C}$ NMR spectrum of compound $21\left(100 \mathrm{MHz}, \mathrm{CDCl}_{3}\right)$

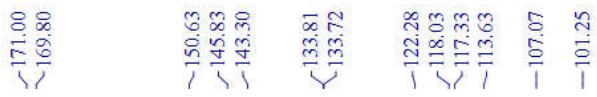

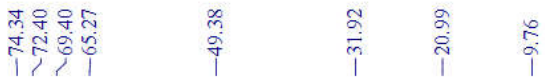<smiles>COc1c(C[C@@H](COC(C)=O)NC(=O)CO[Na])cc2c(c1C)OCO2</smiles>

21

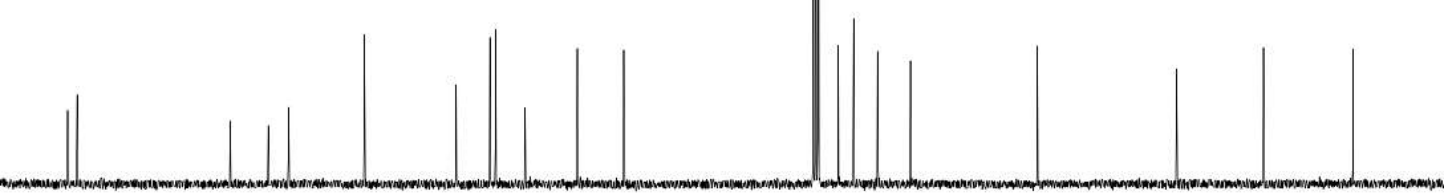

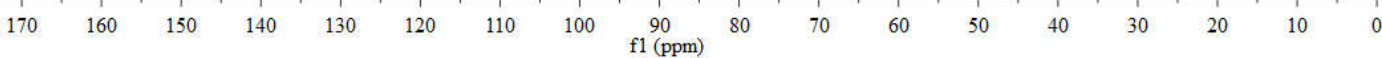


${ }^{1} \mathrm{H}$ NMR spectrum of compound $22 \mathrm{a}\left(400 \mathrm{MHz}, \mathrm{CDCl}_{3}\right)$

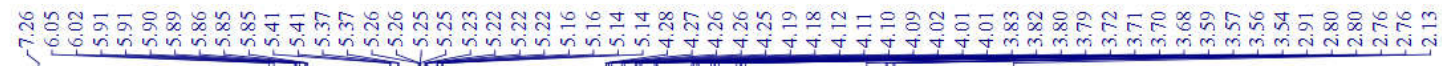<smiles>COc1c2c(c3c(c1OC)OCO3)[C@@H](CO[Co])N[C@H](CO)C2</smiles>

$22 a$

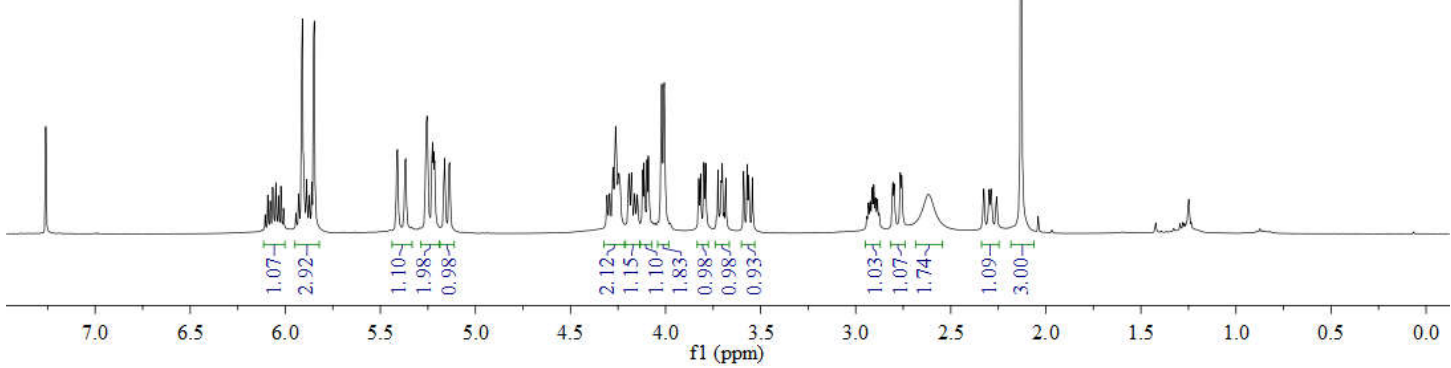

${ }^{13} \mathrm{C}$ NMR spectrum of compound 22a (100 $\left.\mathrm{MHz}, \mathrm{CDCl}_{3}\right)$

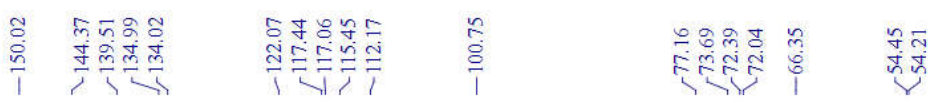<smiles>COc1c(C)c2c(c3c1OCO3)[C@H](COCCOCCO)N[C@H](CO)C2</smiles>

$22 a$

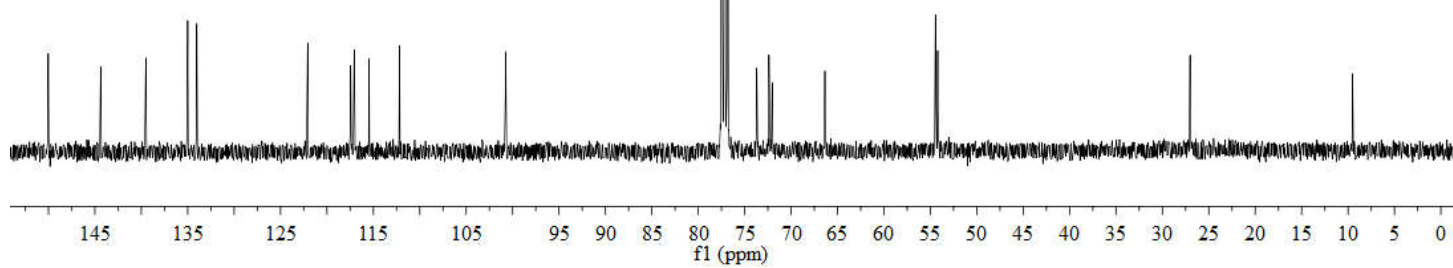


${ }^{1} \mathrm{H}$ NMR spectrum of compound $22 \mathrm{~b}\left(400 \mathrm{MHz}, \mathrm{CDCl}_{3}\right)$

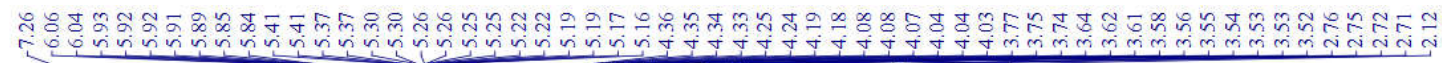<smiles>COc1c(C)c2c(c3c1C[C@@H](CO)N[C@@H]3COCO)OCO2</smiles>

22b

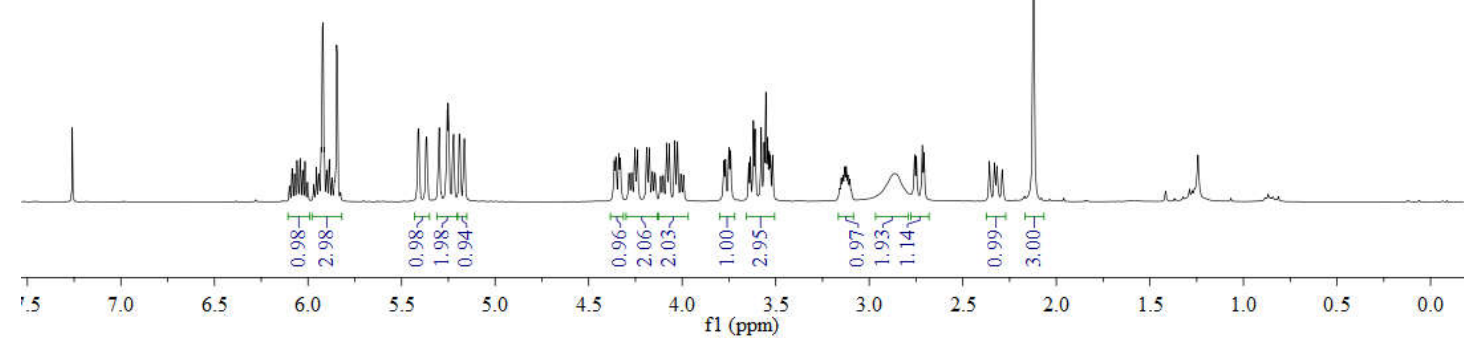

${ }^{13} \mathrm{C}$ NMR spectrum of compound $22 \mathrm{~b}\left(100 \mathrm{MHz}, \mathrm{CDCl}_{3}\right)$

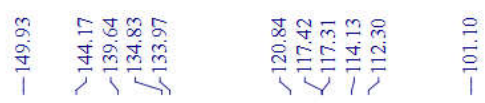

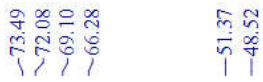<smiles>CCOC[C@H]1N[C@H](CO)Cc2c(OC)c(C)c3c(c21)OCO3</smiles>

22b

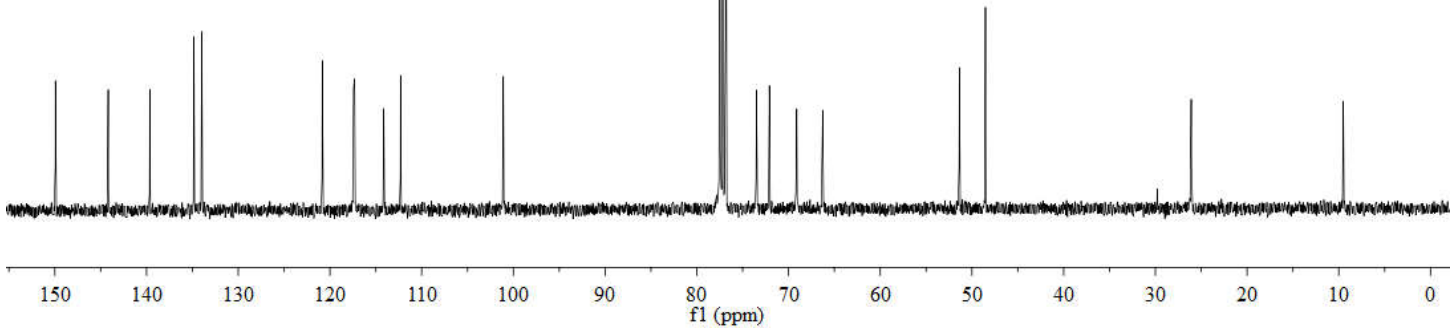


${ }^{1} \mathrm{H}$ NMR spectrum of compound $23\left(400 \mathrm{MHz}, \mathrm{CDCl}_{3}\right)$

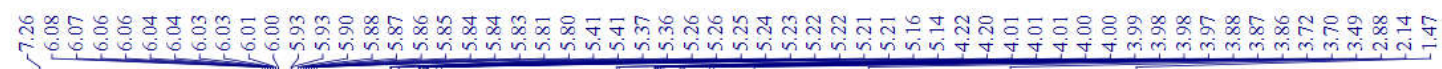

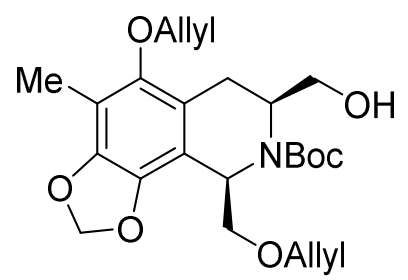

23

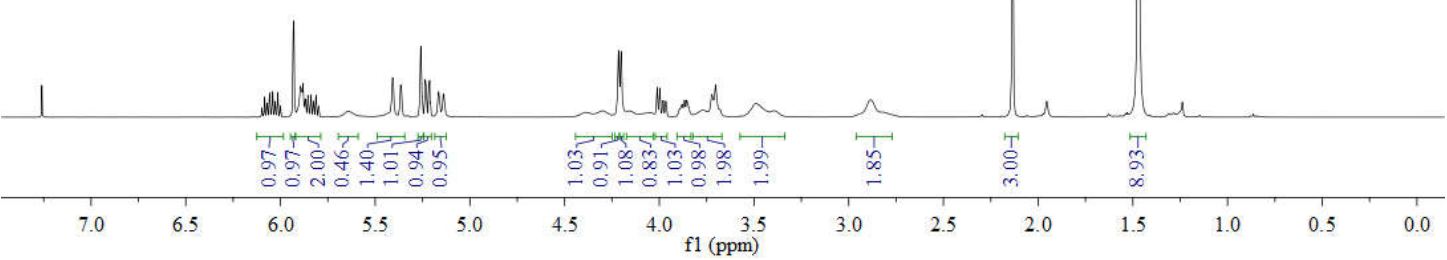

${ }^{13} \mathrm{C}$ NMR spectrum of compound $23\left(100 \mathrm{MHz}, \mathrm{CDCl}_{3}\right)$

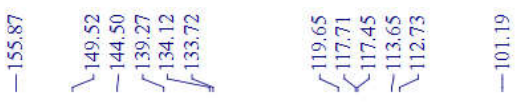

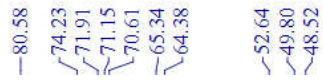

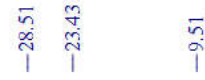<smiles>COc1c(C)c2c(c3c1OCO3)[C@@H](COCCOCCO)[C@H](CO)C2</smiles>

23

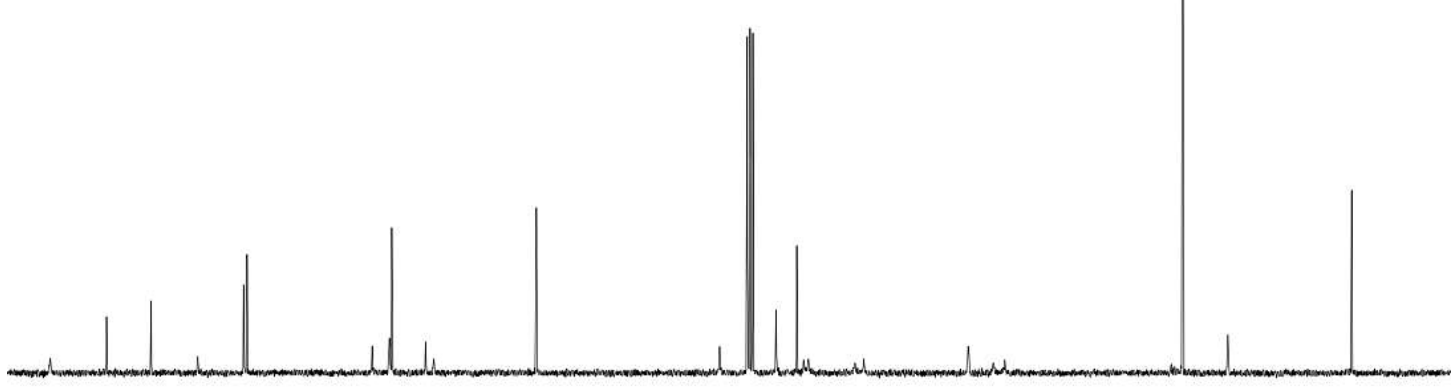

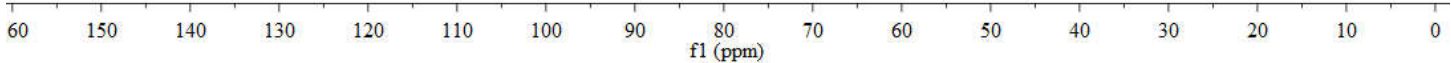


${ }^{1} \mathrm{H}$ NMR spectrum of compound $24\left(100 \mathrm{MHz}, \mathrm{CDCl}_{3}\right)$

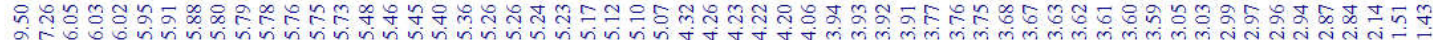

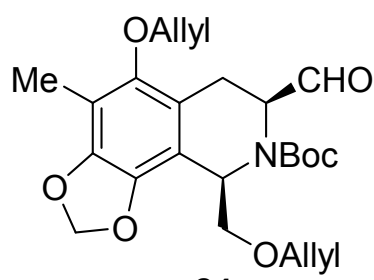

24

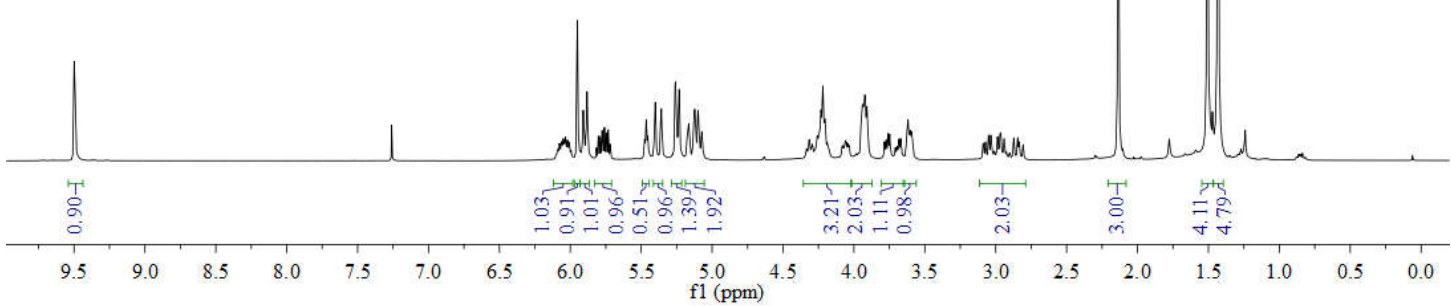

${ }^{13} \mathrm{C}$ NMR spectrum of compound $24\left(100 \mathrm{MHz}, \mathrm{CDCl}_{3}\right)$

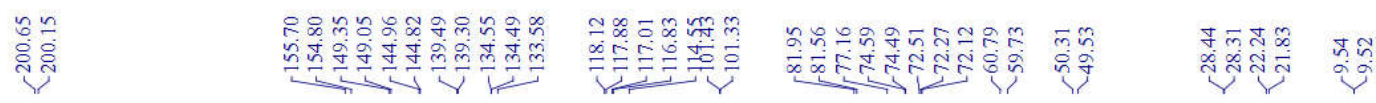<smiles>COCC1c2c(c(OC)c(C)c3c2OCO3)C[C@H](C=O)[NH+]1C(=O)O</smiles>

24

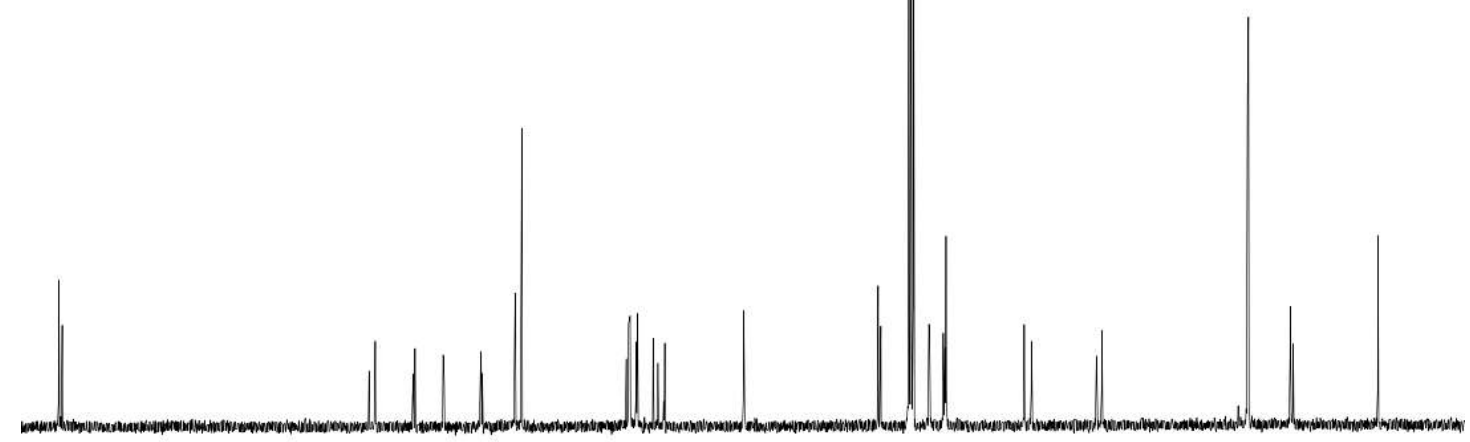

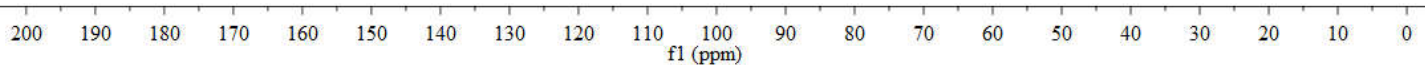


${ }^{1} \mathrm{H}$ NMR spectrum of compound $25\left(400 \mathrm{MHz}, \mathrm{CDCl}_{3}\right)$

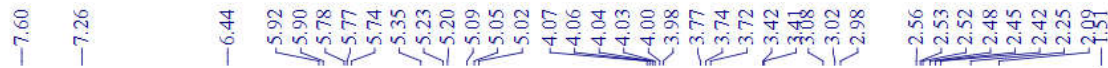

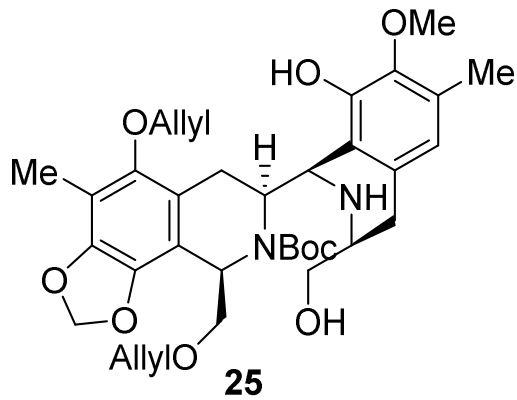

25

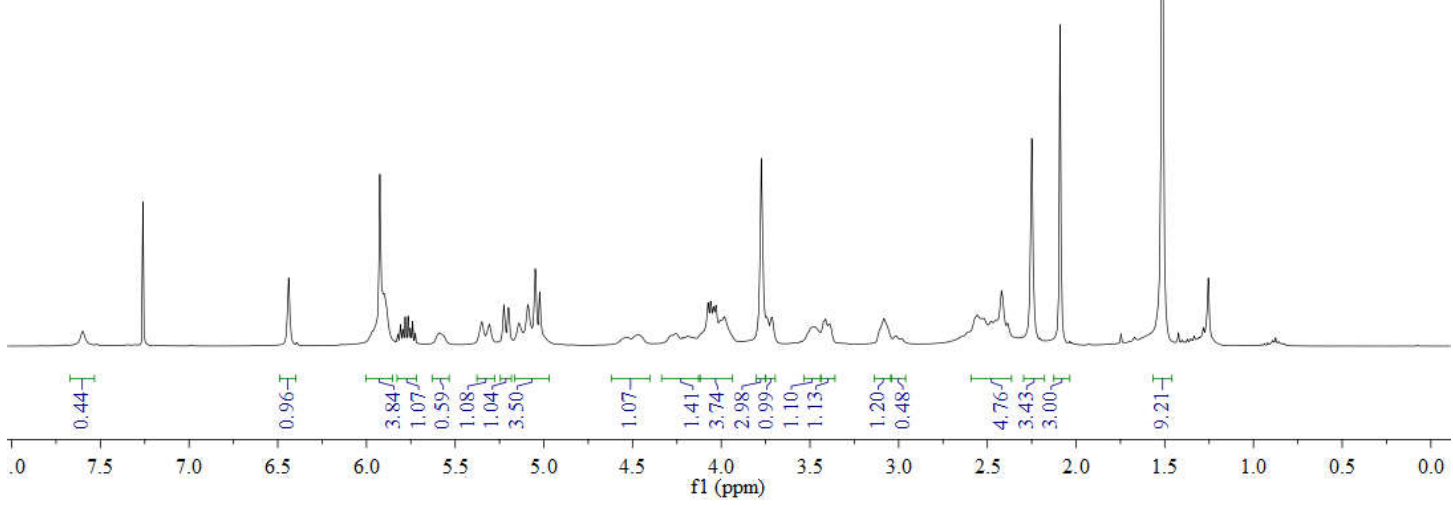

${ }^{13} \mathrm{C}$ NMR spectrum of compound $25\left(100 \mathrm{MHz}, \mathrm{CDCl}_{3}\right)$

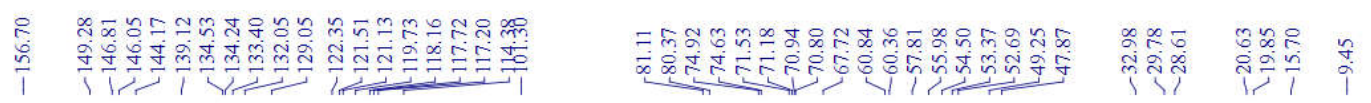

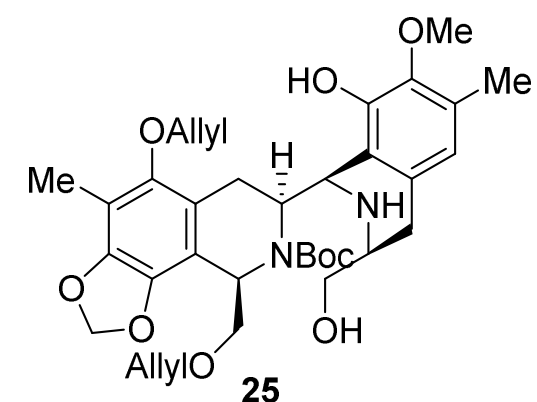

25

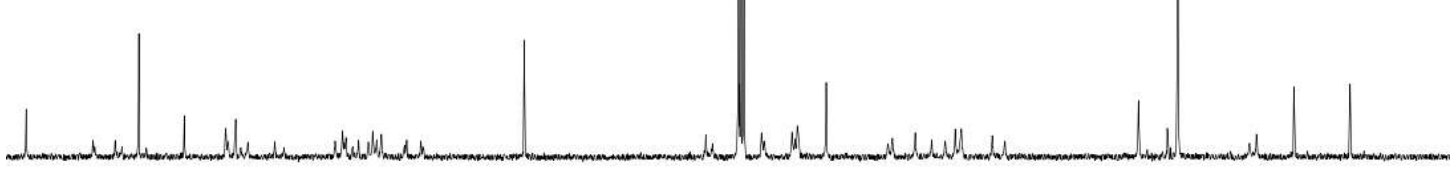

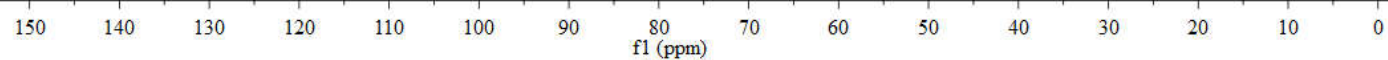


${ }^{1} \mathrm{H}$ NMR spectrum of compound $26\left(400 \mathrm{MHz}, \mathrm{CDCl}_{3}\right)$

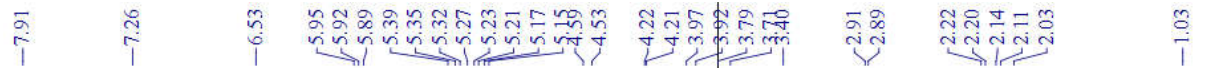

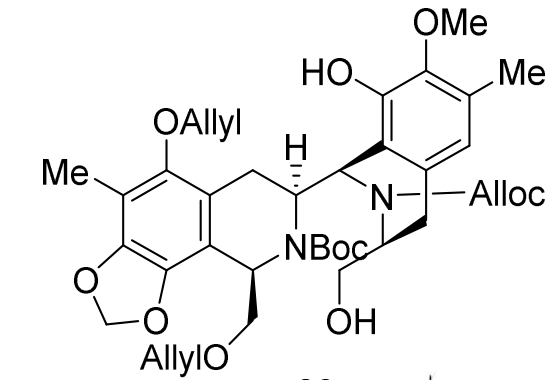

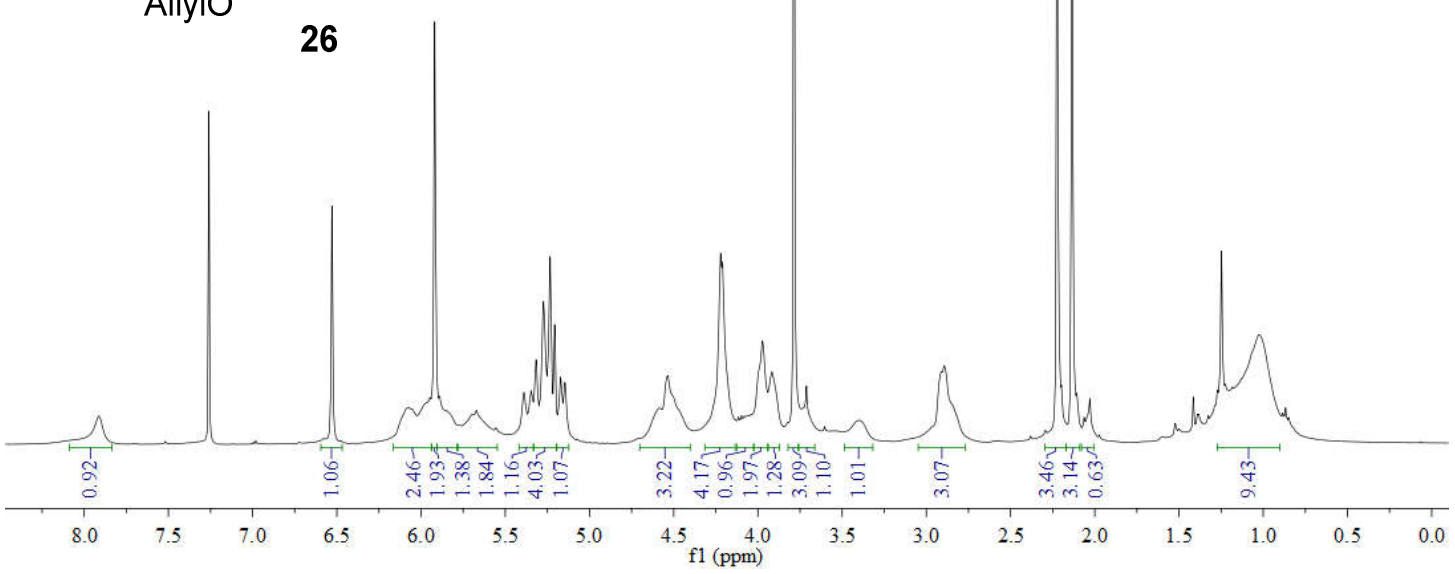

${ }^{13} \mathrm{C}$ NMR spectrum of compound $26\left(100 \mathrm{MHz}, \mathrm{CDCl}_{3}\right)$

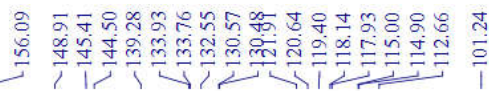

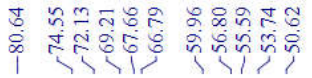

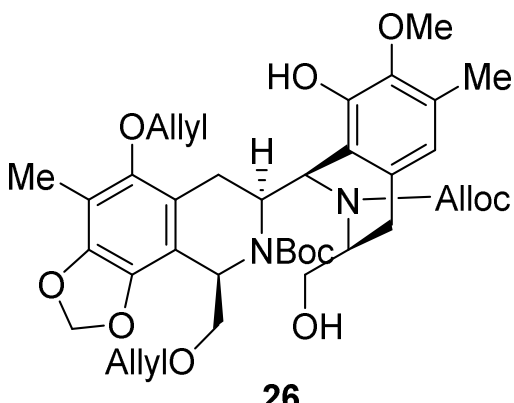

26

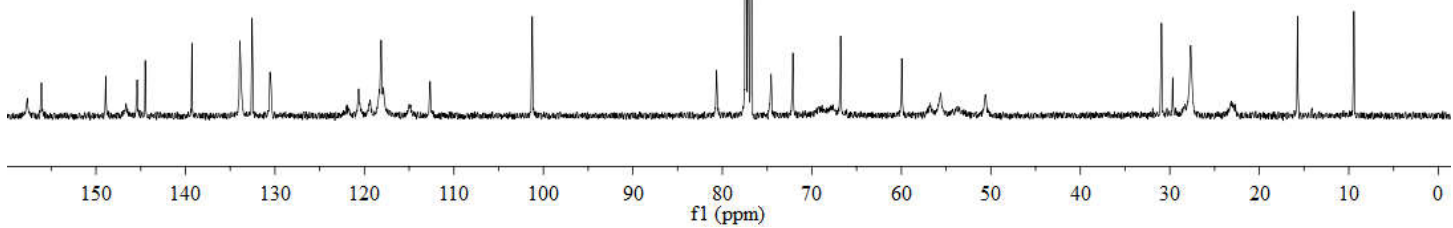


${ }^{1} \mathrm{H}$ NMR spectrum of compound $27\left(400 \mathrm{MHz}, \mathrm{CDCl}_{3}\right)$

过

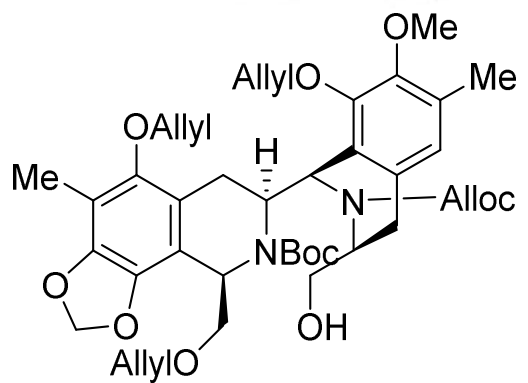

27

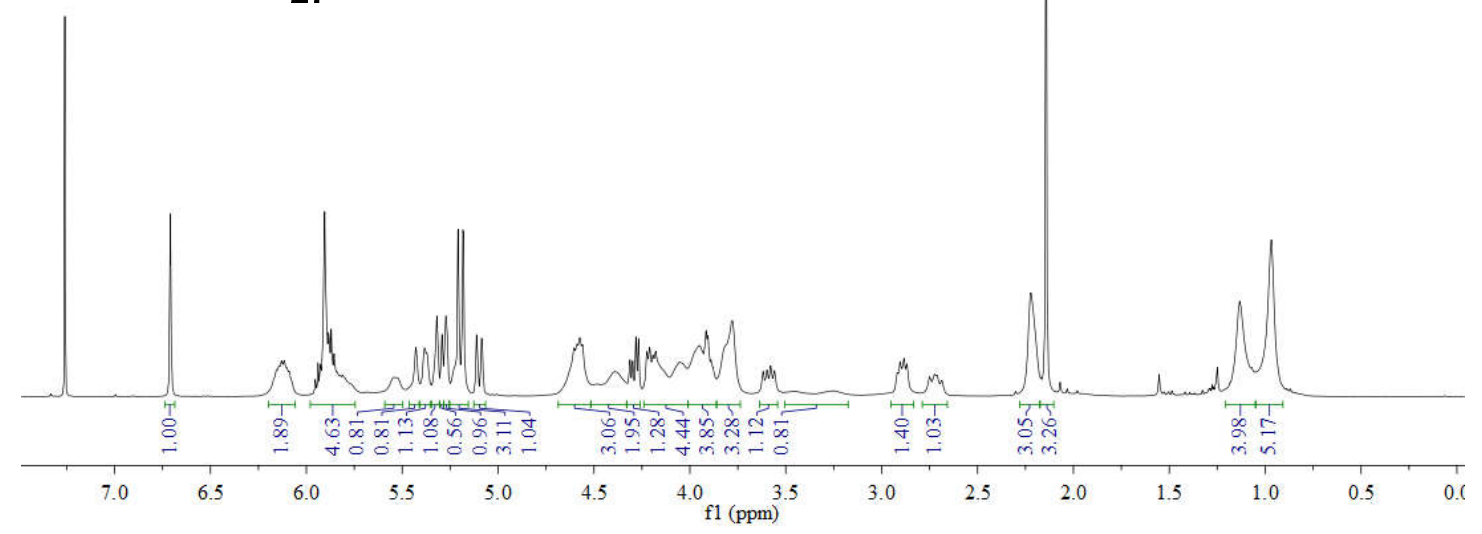

${ }^{13} \mathrm{C}$ NMR spectrum of compound $27\left(100 \mathrm{MHz} \mathrm{CDCl}_{3}\right)$

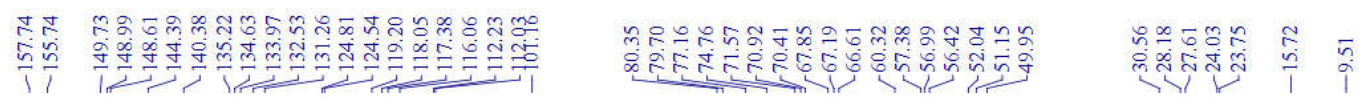

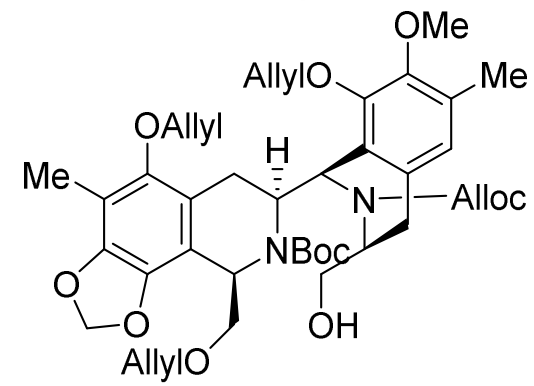

27

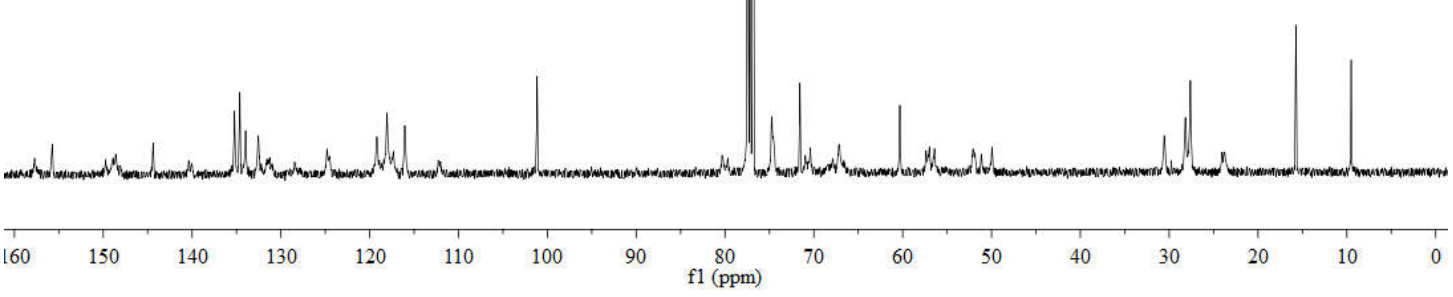


${ }^{1} \mathrm{H}$ NMR spectrum of compound $28\left(400 \mathrm{MHz}, \mathrm{CDCl}_{3}\right)$

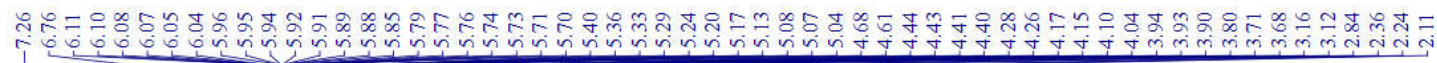

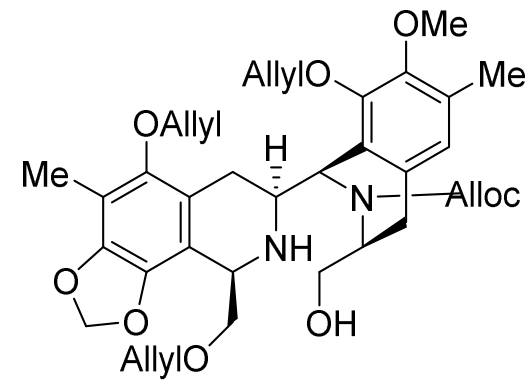

28

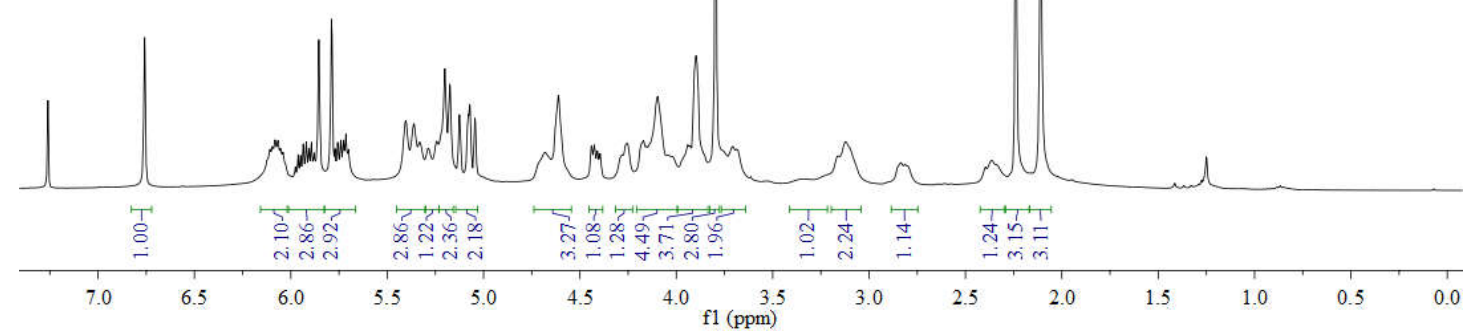

${ }^{13} \mathrm{C}$ NMR spectrum of compound $28\left(100 \mathrm{MHz}, \mathrm{CDCl}_{3}\right)$

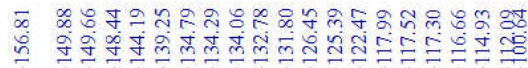

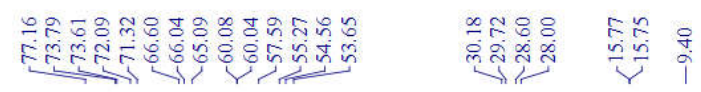

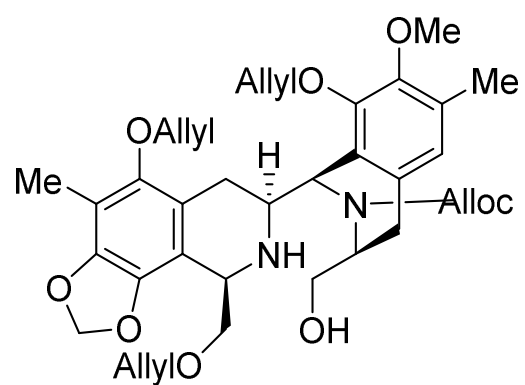

28

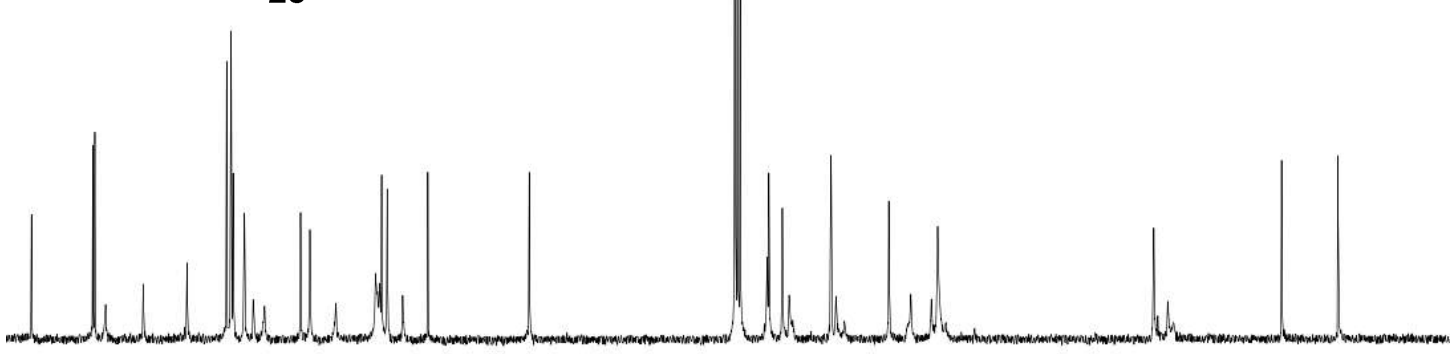

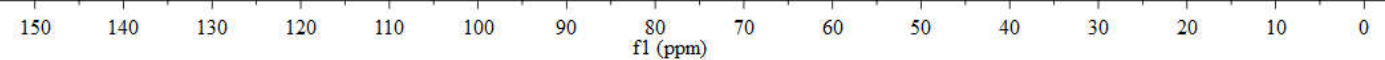


${ }^{1} \mathrm{H}$ NMR spectrum of compound $29\left(400 \mathrm{MHz}, \mathrm{CDCl}_{3}\right)$

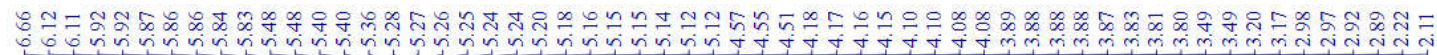

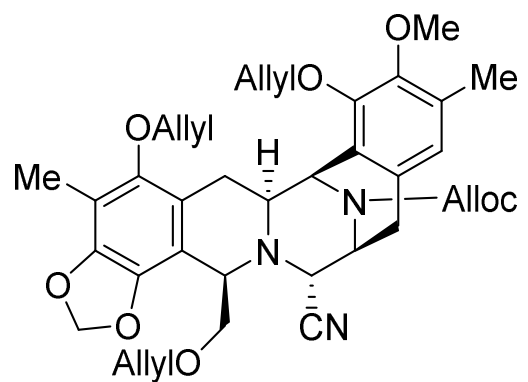

29

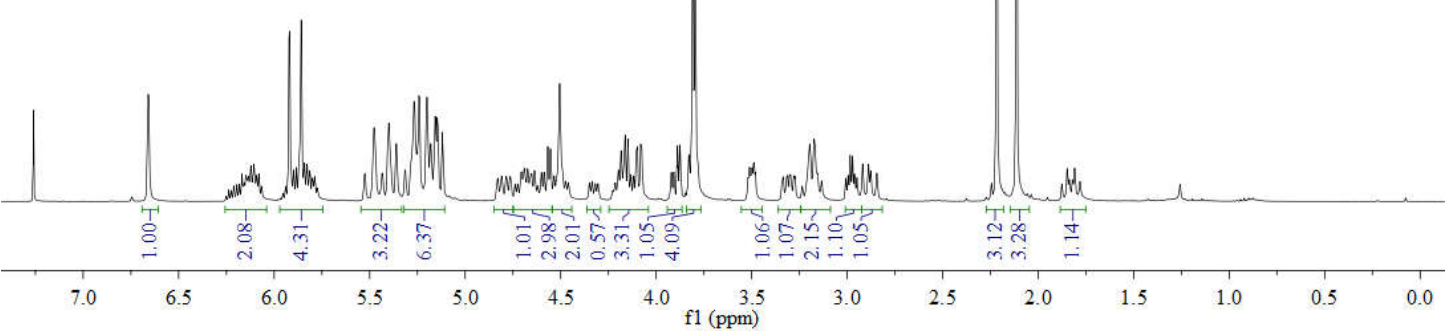

${ }^{13} \mathrm{C}$ NMR spectrum of compound $29\left(100 \mathrm{MHz}, \mathrm{CDCl}_{3}\right)$

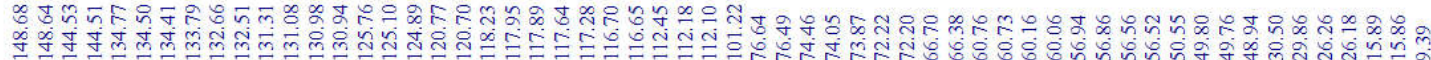

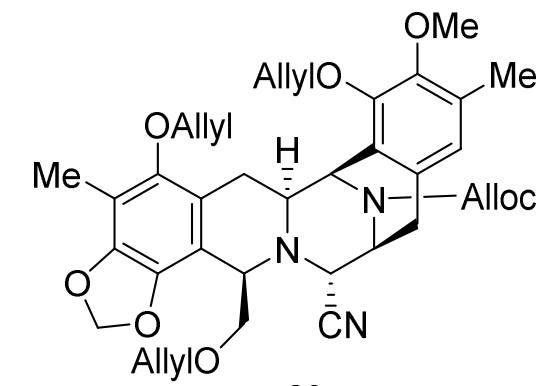

29

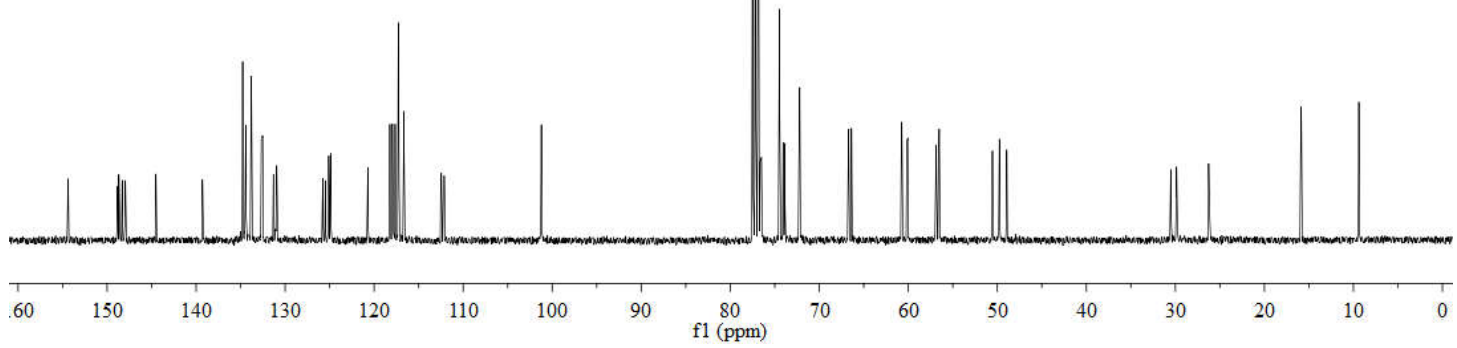


${ }^{1} \mathrm{H}$ NMR spectrum of compound $30\left(400 \mathrm{MHz}, \mathrm{CDCl}_{3}\right)$

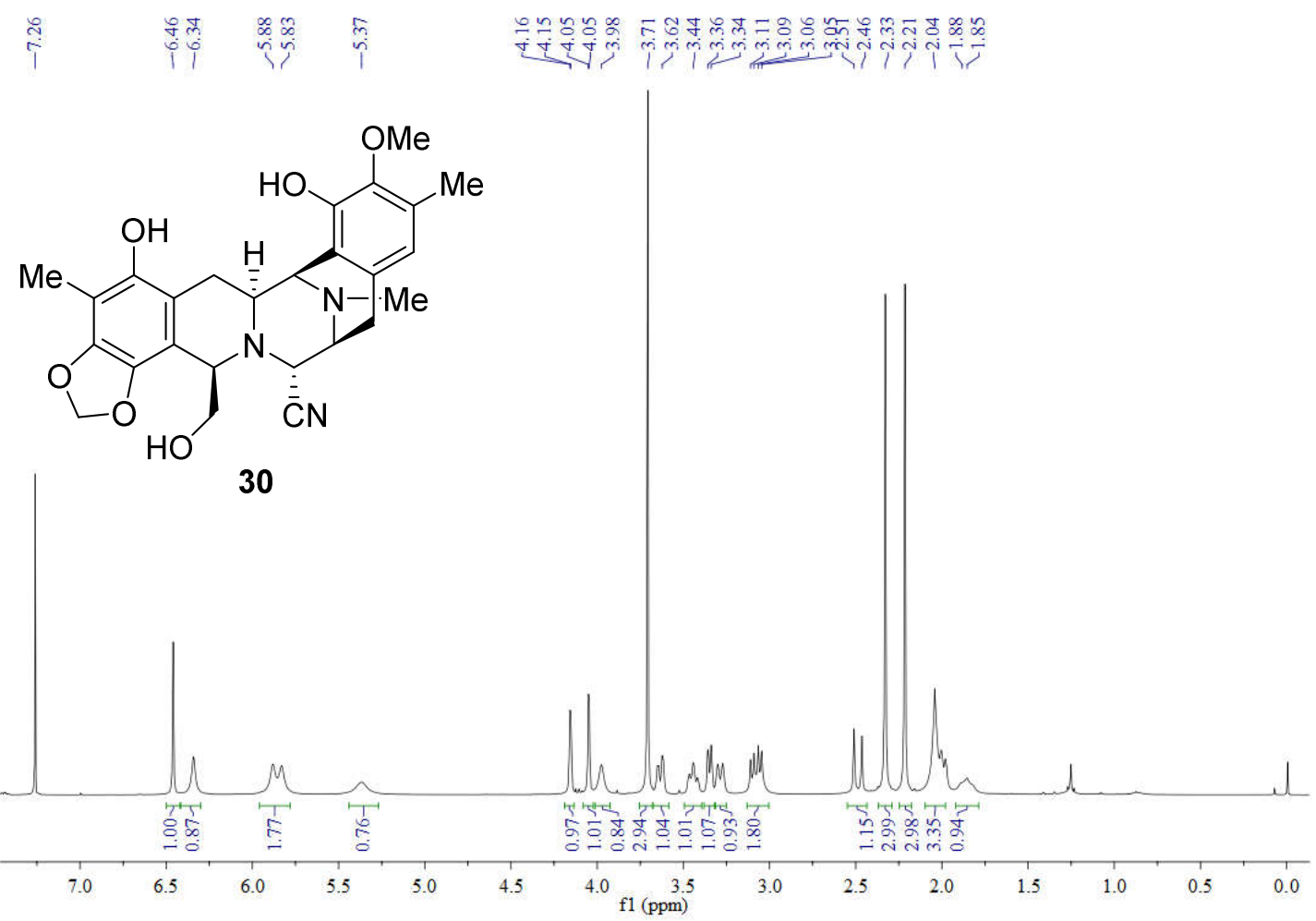

${ }^{13} \mathrm{C}$ NMR spectrum of compound $30\left(100 \mathrm{MHz}, \mathrm{CDCl}_{3}\right)$

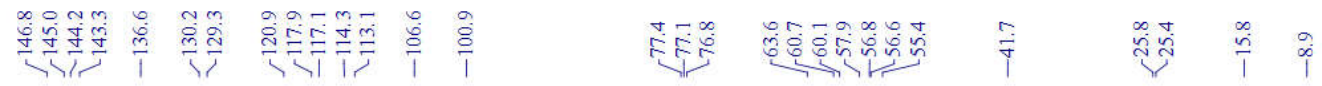

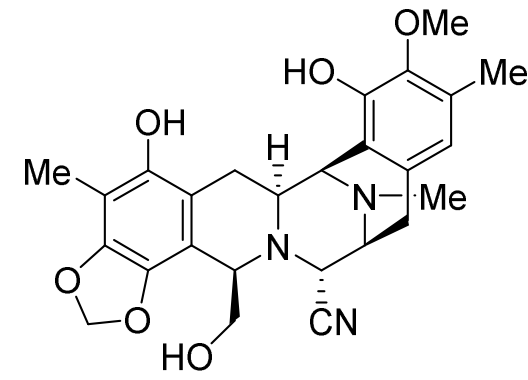

30

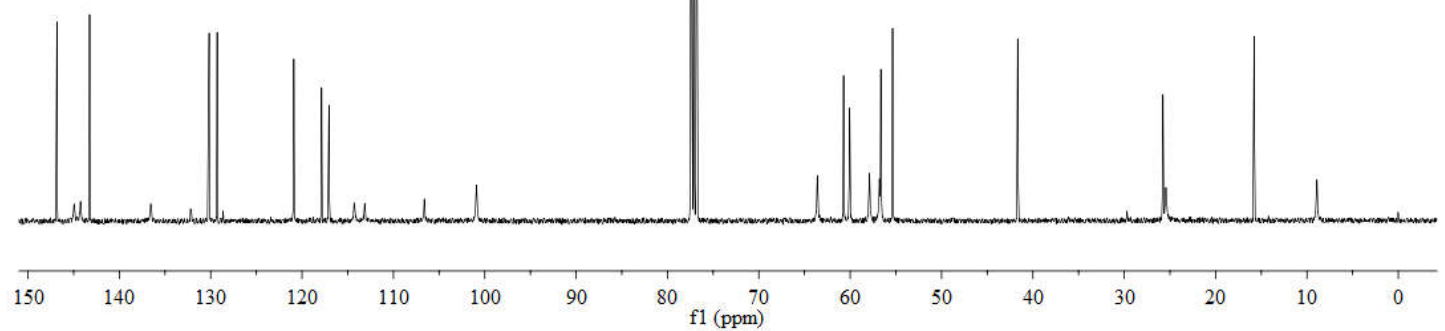


${ }^{1} \mathrm{H}$ NMR spectrum of compound $38\left(400 \mathrm{MHz}, \mathrm{CDCl}_{3}\right)$

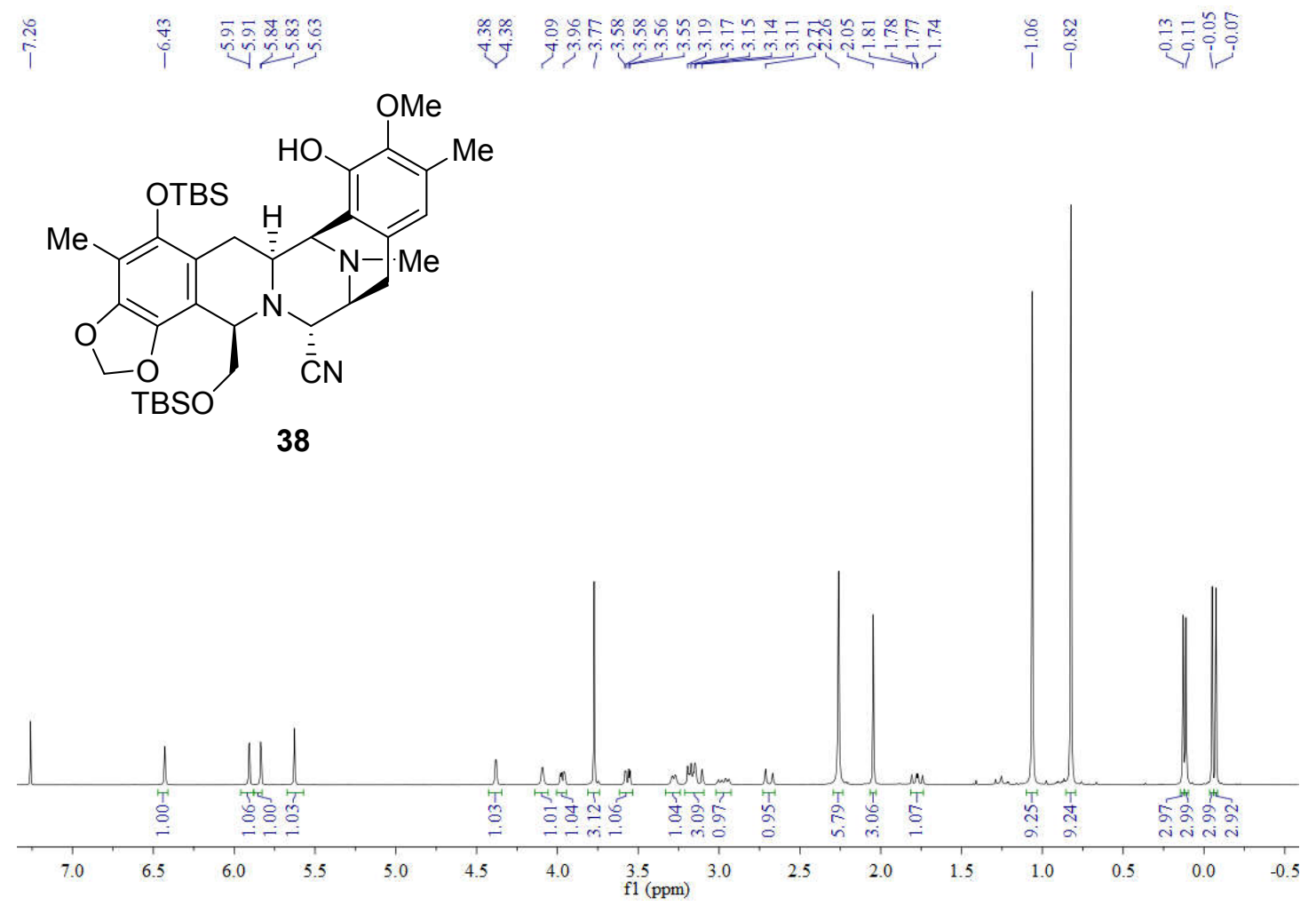

${ }^{13} \mathrm{C}$ NMR spectrum of compound $38\left(100 \mathrm{MHz}, \mathrm{CDCl}_{3}\right)$

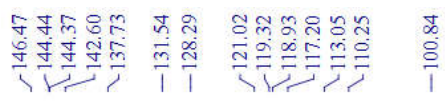

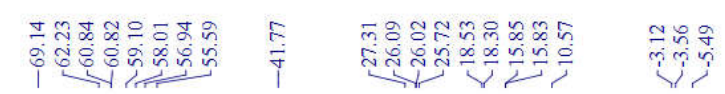<smiles>COc1ccc(C(C)C)c(O)c1O</smiles>

38

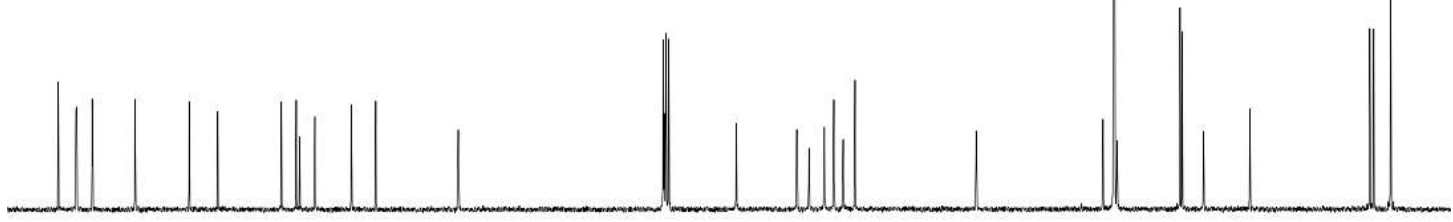

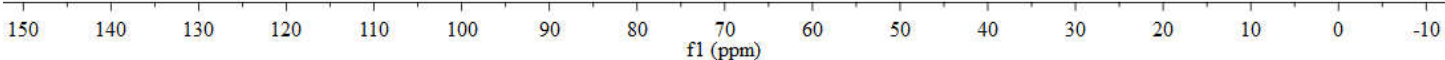


${ }^{1} \mathrm{H}$ NMR spectrum of compound $39\left(400 \mathrm{MHz}, \mathrm{CDCl}_{3}\right)$

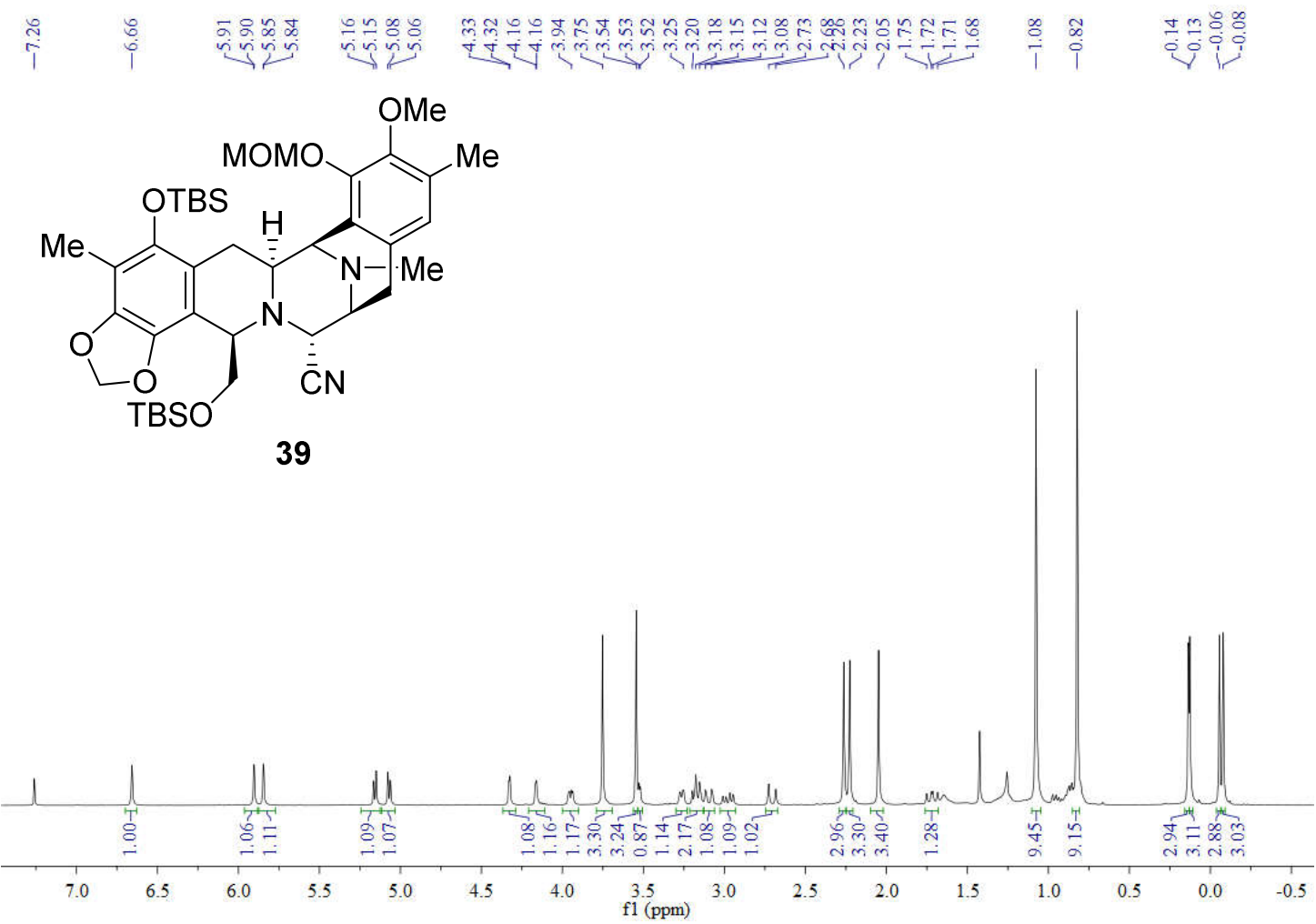

${ }^{13} \mathrm{C}$ NMR spectrum of compound $39\left(100 \mathrm{MHz}, \mathrm{CDCl}_{3}\right)$

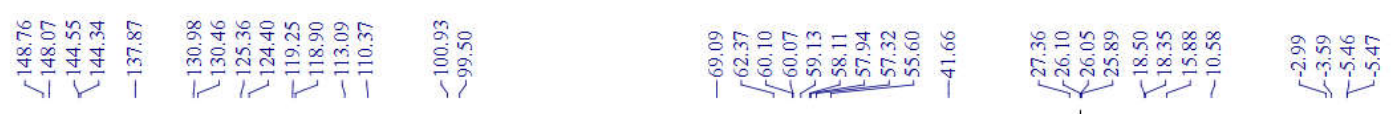

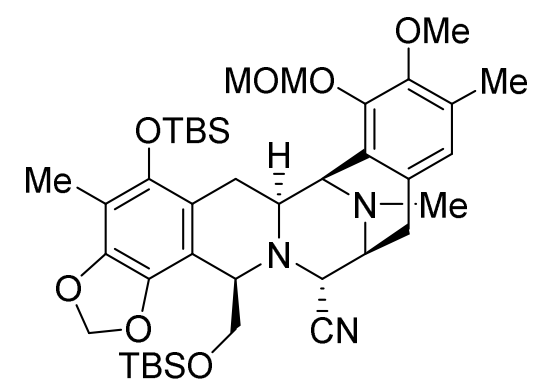

39

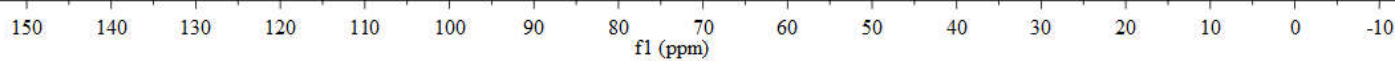


${ }^{1} \mathrm{H}$ NMR spectrum of compound $40\left(400 \mathrm{MHz}, \mathrm{CDCl}_{3}\right)$

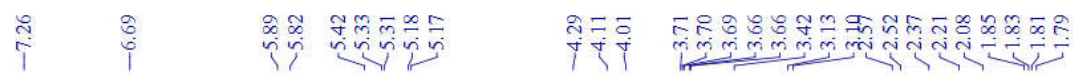

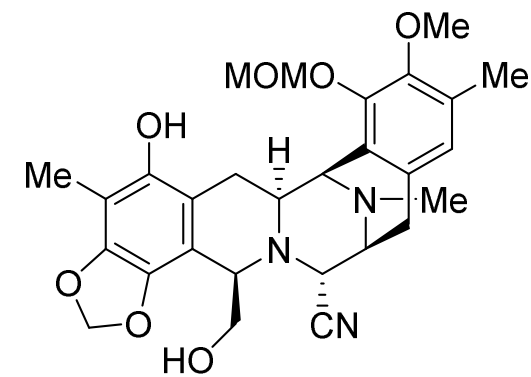

40
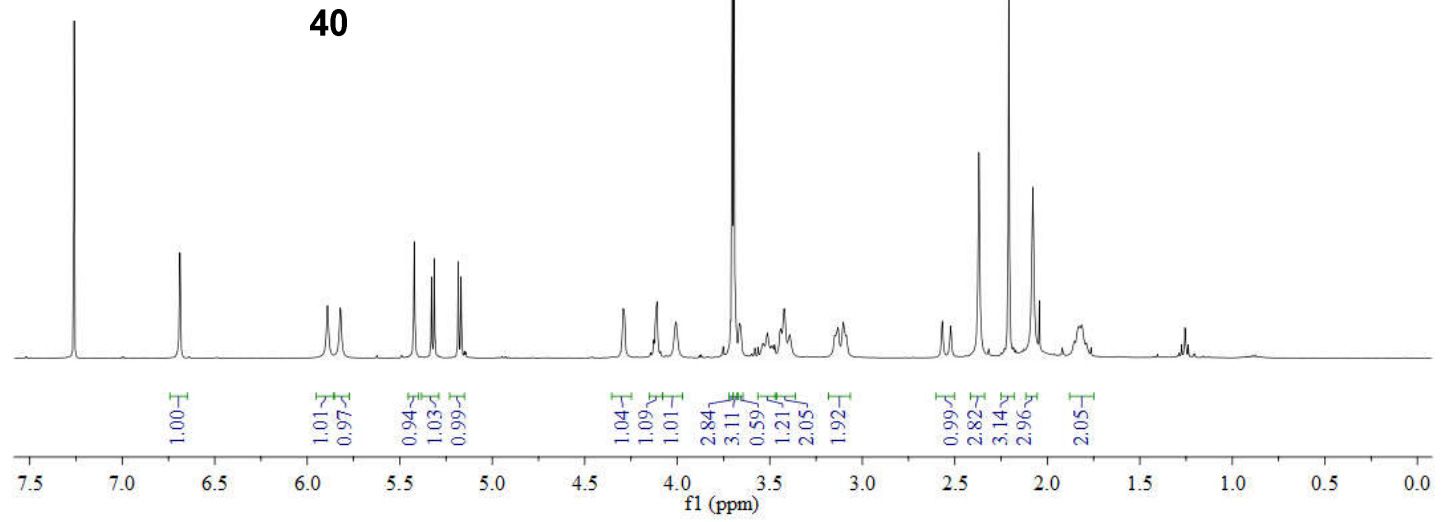

${ }^{13} \mathrm{C}$ NMR spectrum of compound $40\left(100 \mathrm{MHz}, \mathrm{CDCl}_{3}\right)$

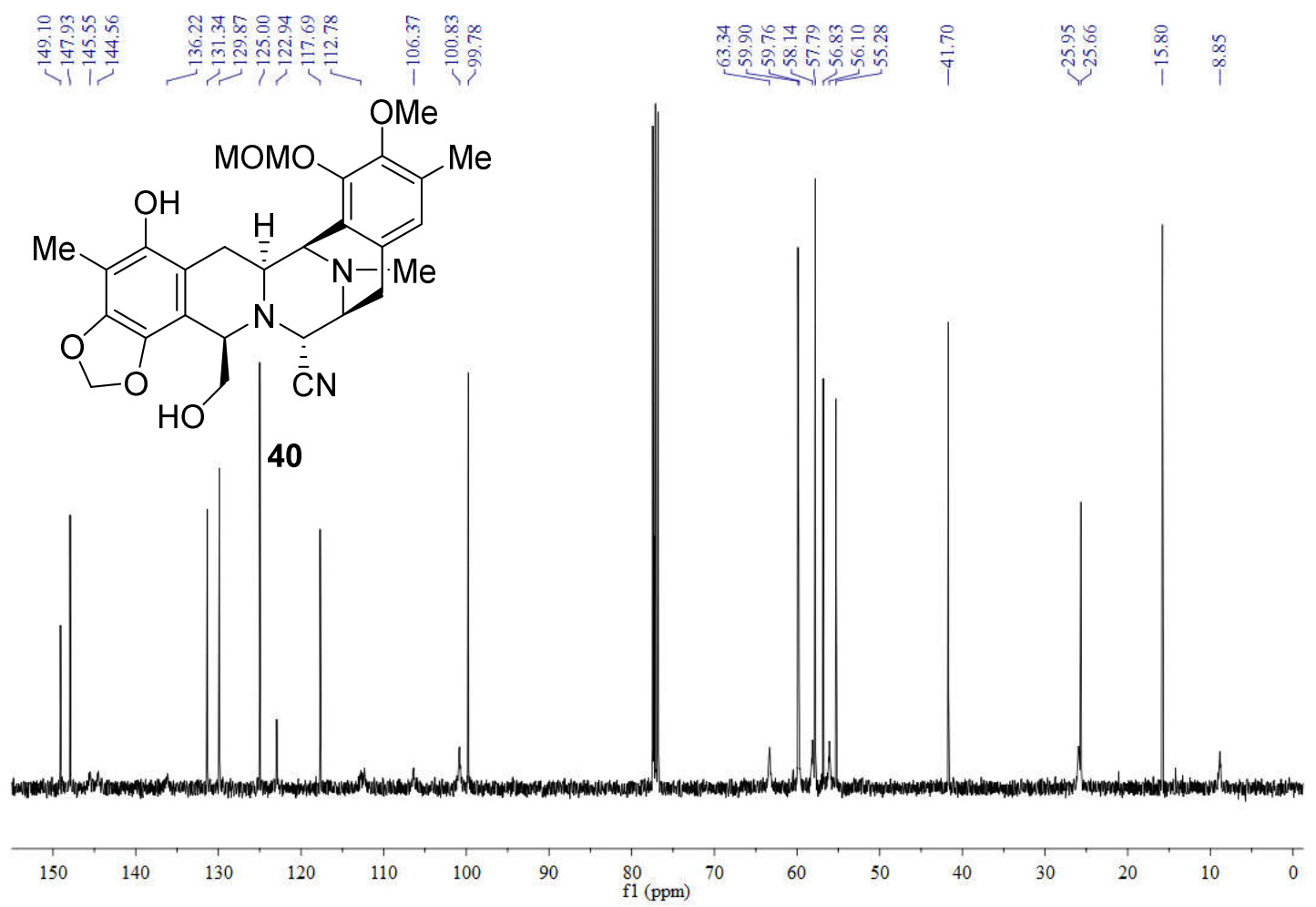


${ }^{1} \mathrm{H}$ NMR spectrum of compound $42\left(400 \mathrm{MHz}, \mathrm{CDCl}_{3}\right)$

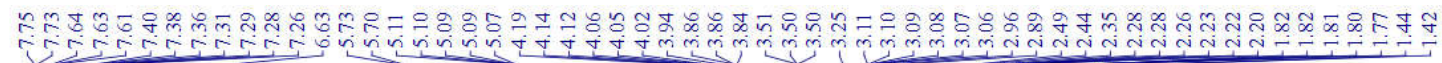

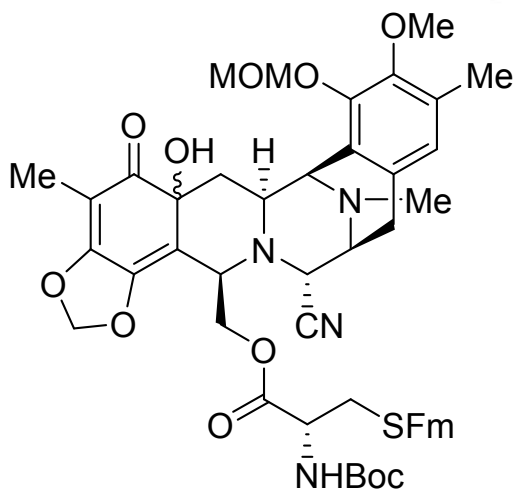

42 less polar isomer

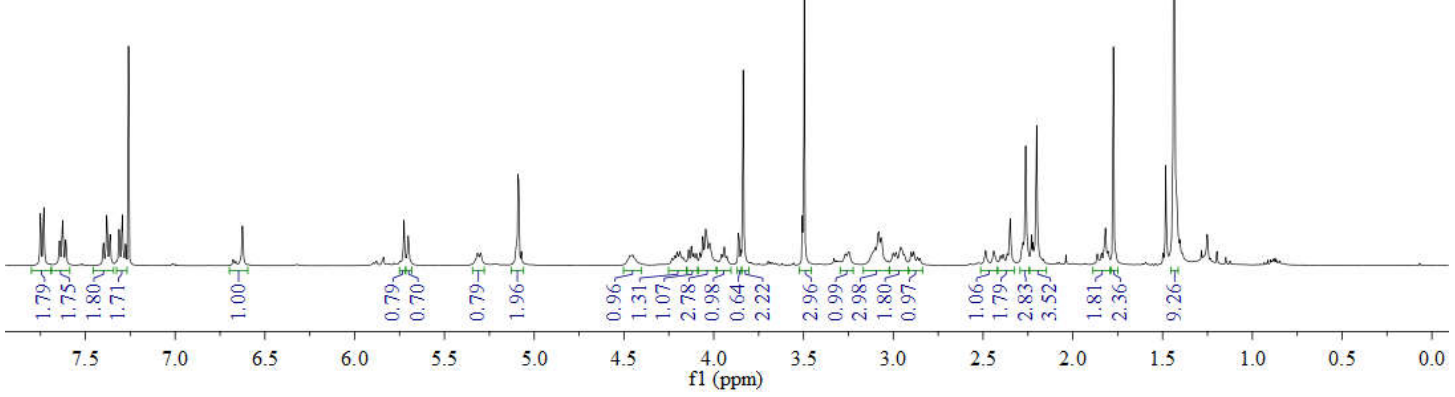

${ }^{13} \mathrm{C}$ NMR spectrum of compound $42\left(100 \mathrm{MHz}, \mathrm{CDCl}_{3}\right)$

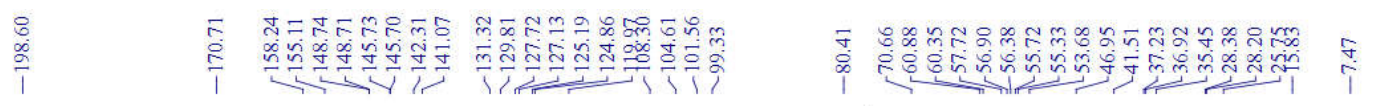

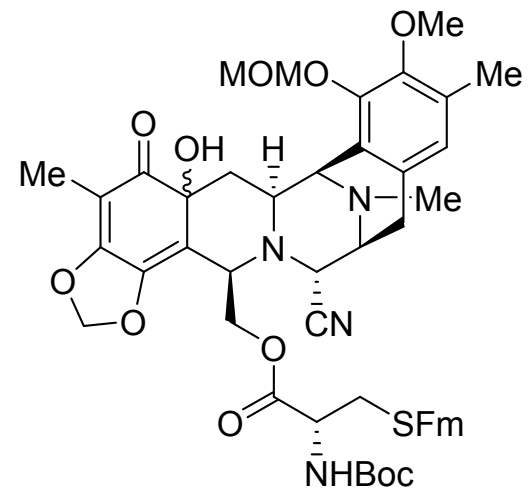

42 less polar isomer

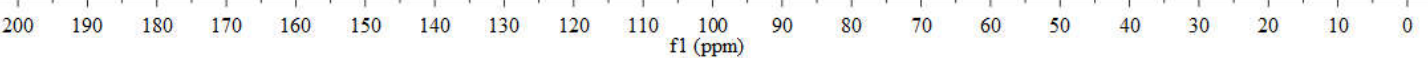


${ }^{1} \mathrm{H}$ NMR spectrum of compound $42\left(400 \mathrm{MHz}, \mathrm{CDCl}_{3}\right)$

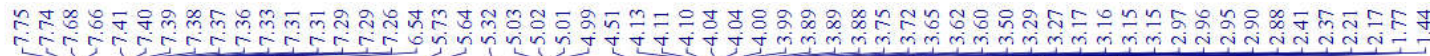

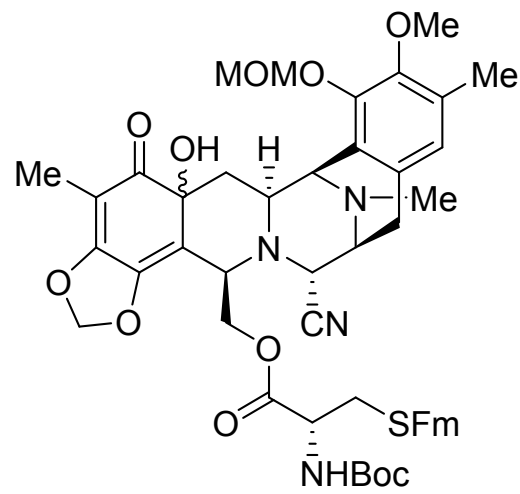

42 more polar isomer

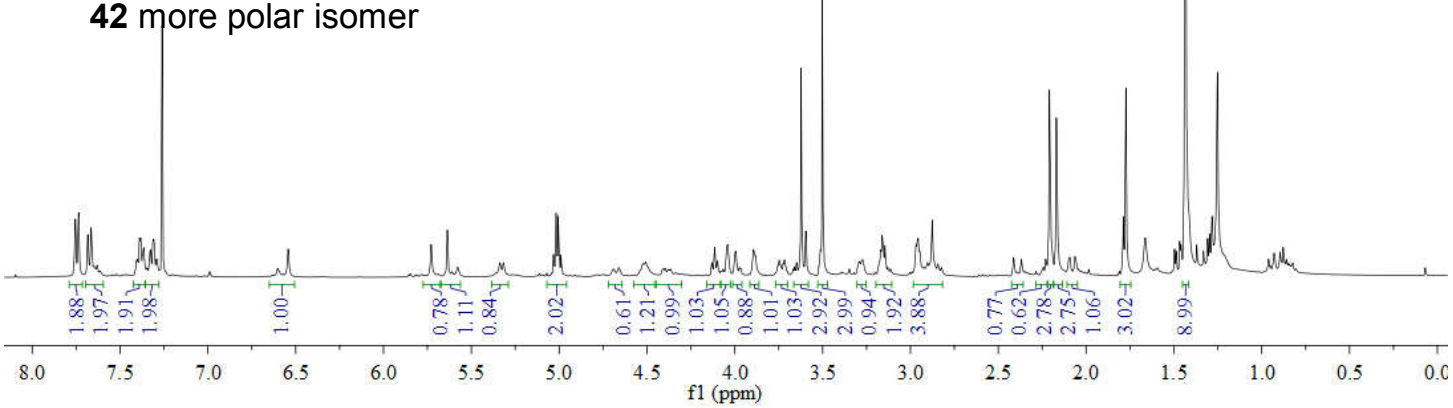

${ }^{13} \mathrm{C}$ NMR spectrum of compound $42\left(100 \mathrm{MHz}, \mathrm{CDCl}_{3}\right)$

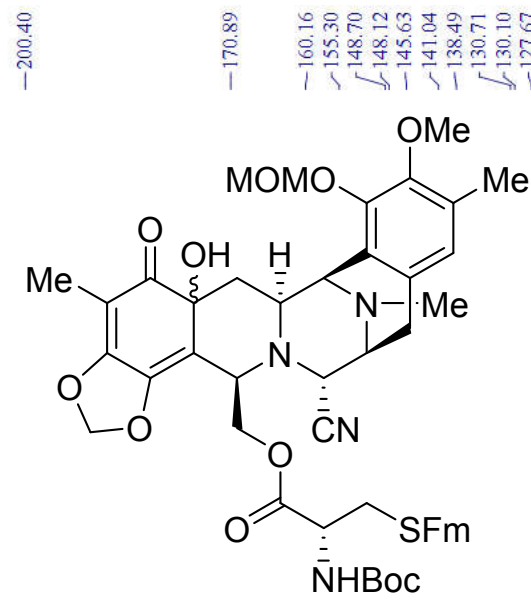

42 more polar isomer

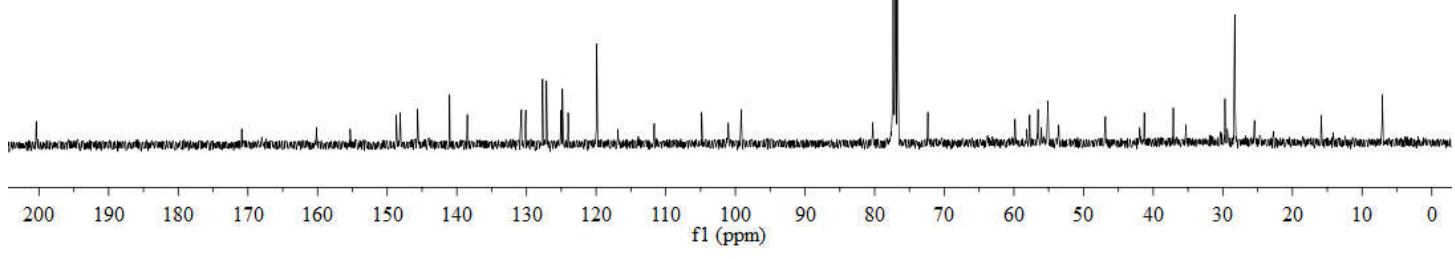


${ }^{1} \mathrm{H}$ NMR spectrum of compound $43\left(400 \mathrm{MHz}, \mathrm{CDCl}_{3}\right)$

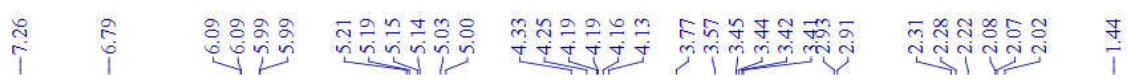

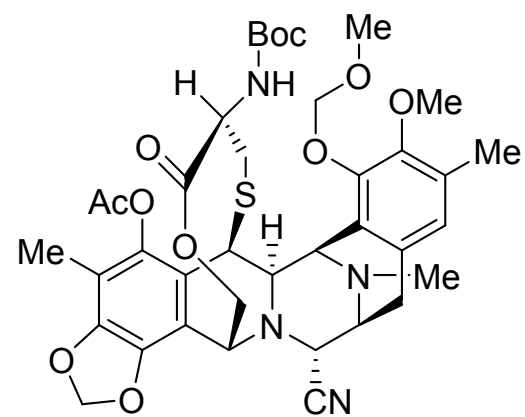

43

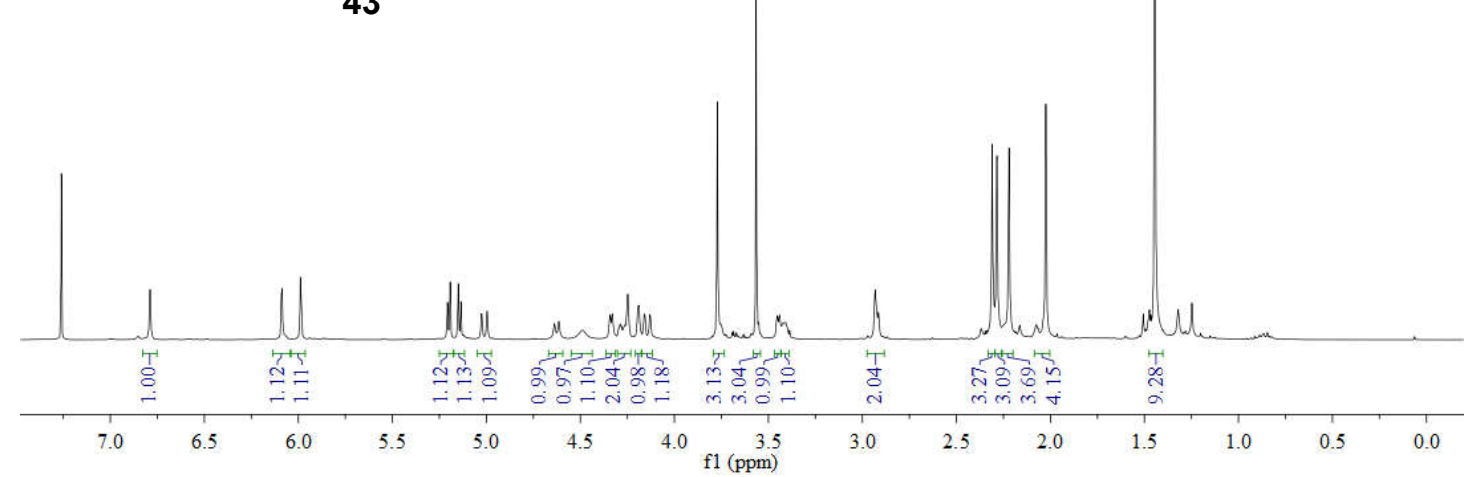

${ }^{13} \mathrm{C}$ NMR spectrum of compound $43\left(100 \mathrm{MHz}, \mathrm{CDCl}_{3}\right)$

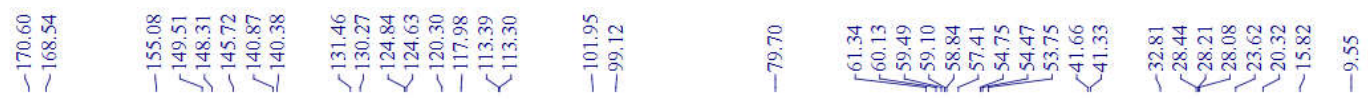

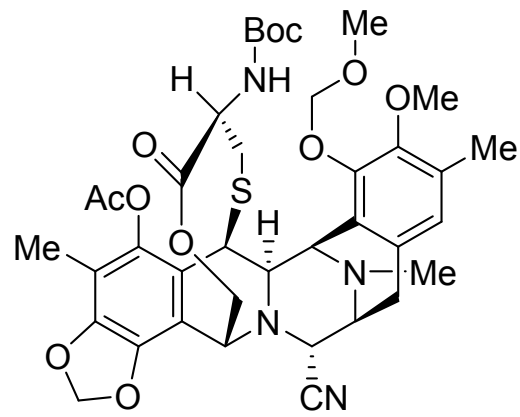

43

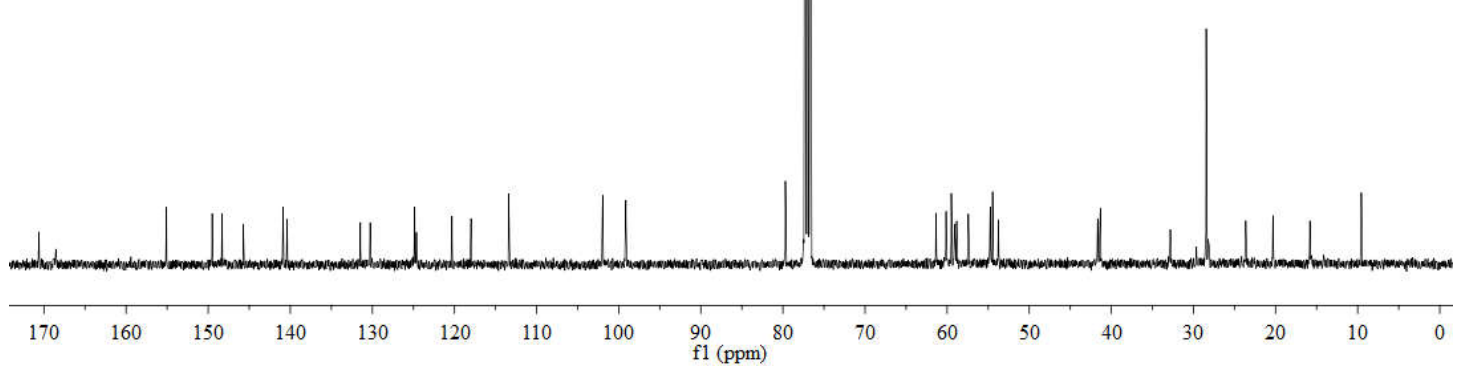


${ }^{1} \mathrm{H}$ NMR spectrum of compound $44\left(400 \mathrm{MHz}, \mathrm{CDCl}_{3}\right)$

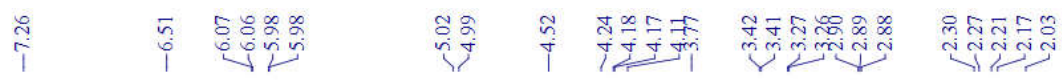

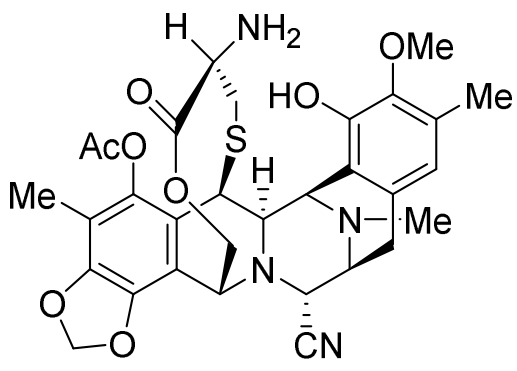

44

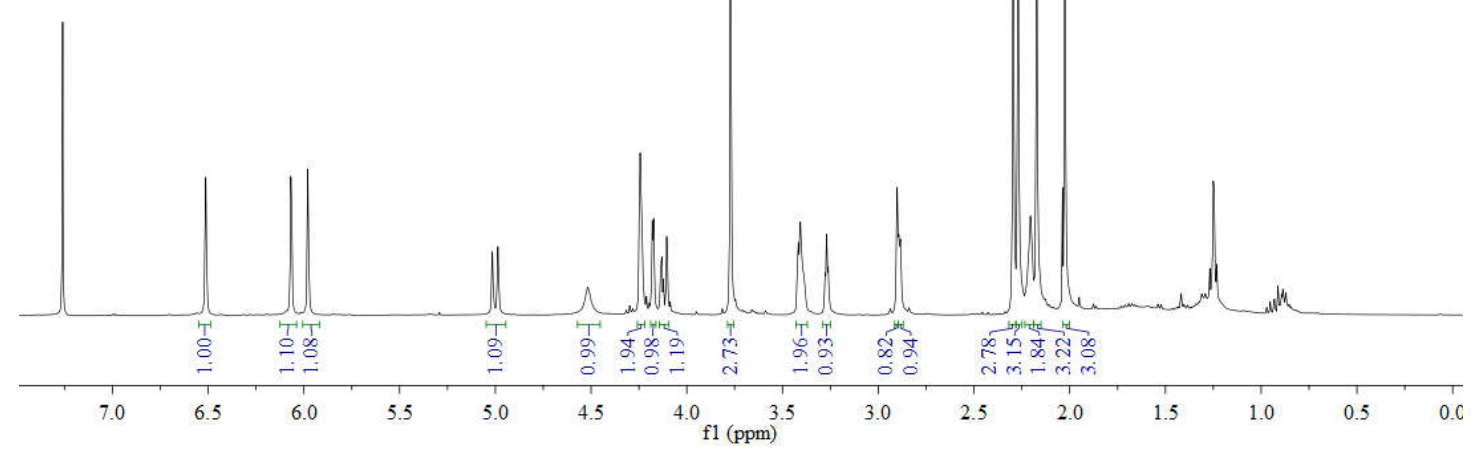

${ }^{13} \mathrm{C}$ NMR spectrum of compound $44\left(100 \mathrm{MHz}, \mathrm{CDCl}_{3}\right)$

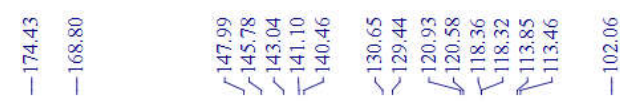

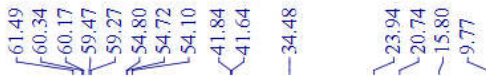

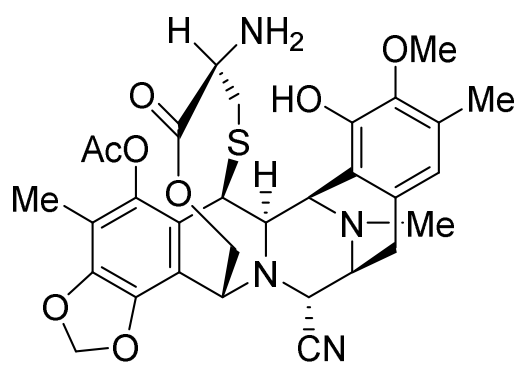

44

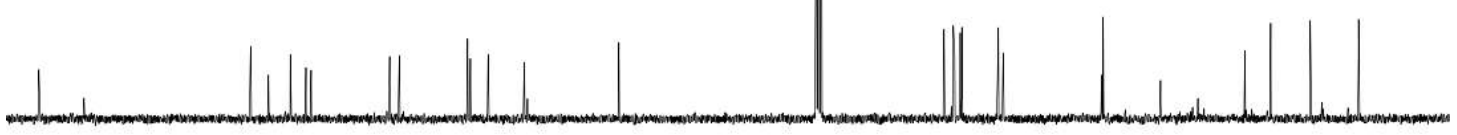

$\begin{array}{llllllllllllllllll}170 & 160 & 150 & 140 & 130 & 120 & 110 & 100 & \begin{array}{c}90 \\ \mathrm{f1}(\mathrm{ppm})\end{array} & 80 & 70 & 60 & 50 & 40 & 30 & 20 & 10 & 0\end{array}$ 
${ }^{1} \mathrm{H}$ NMR spectrum of compound $45\left(400 \mathrm{MHz}, \mathrm{CDCl}_{3}\right)$

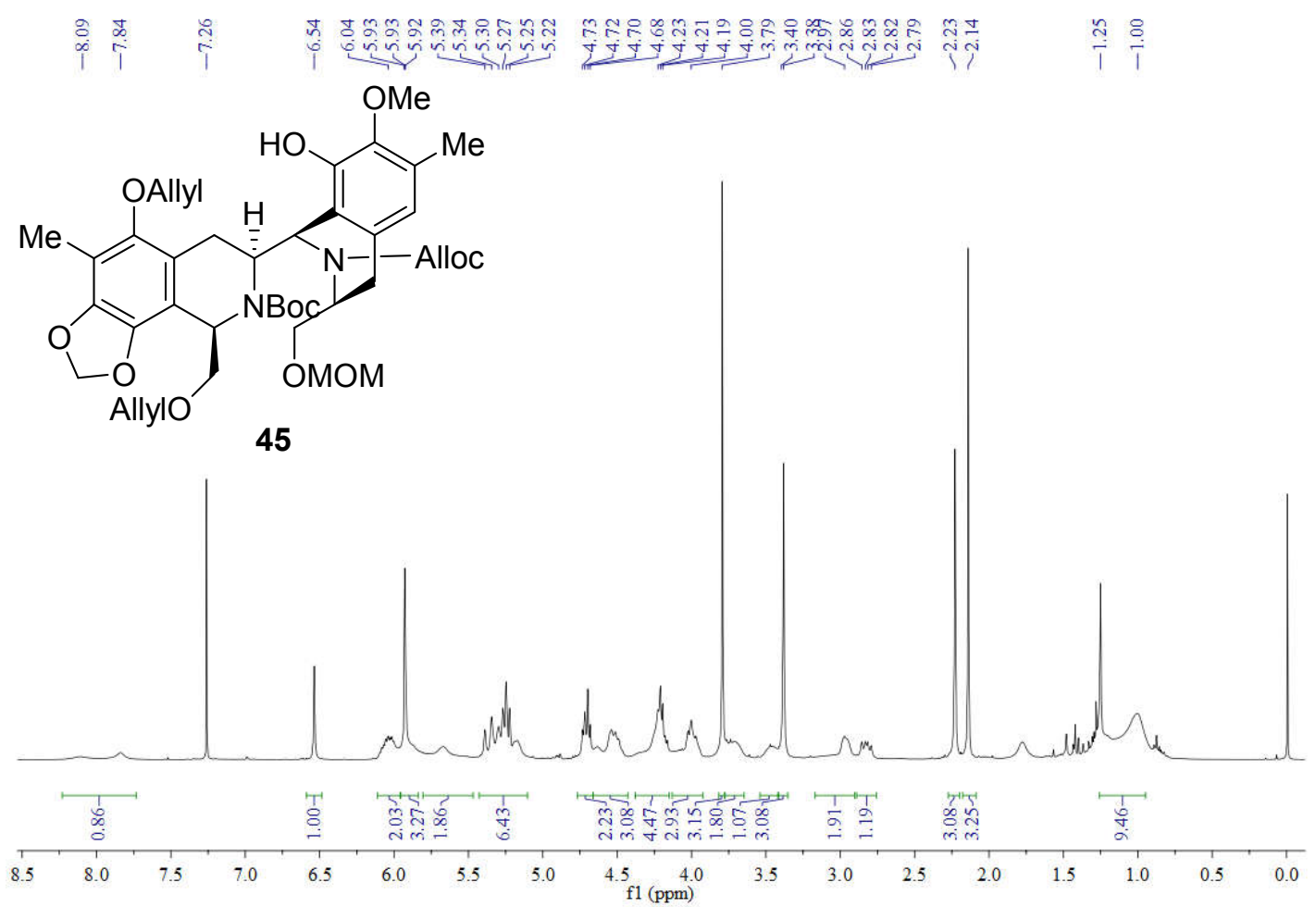

${ }^{1} \mathrm{H}$ NMR spectrum of compound $46\left(400 \mathrm{MHz}, \mathrm{CDCl}_{3}\right)$

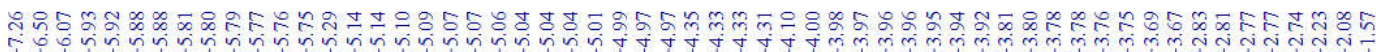

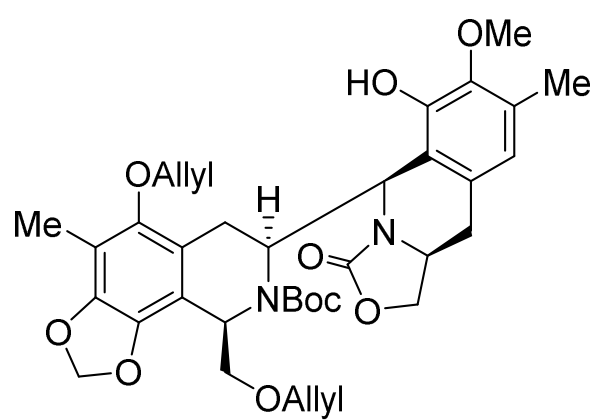

46

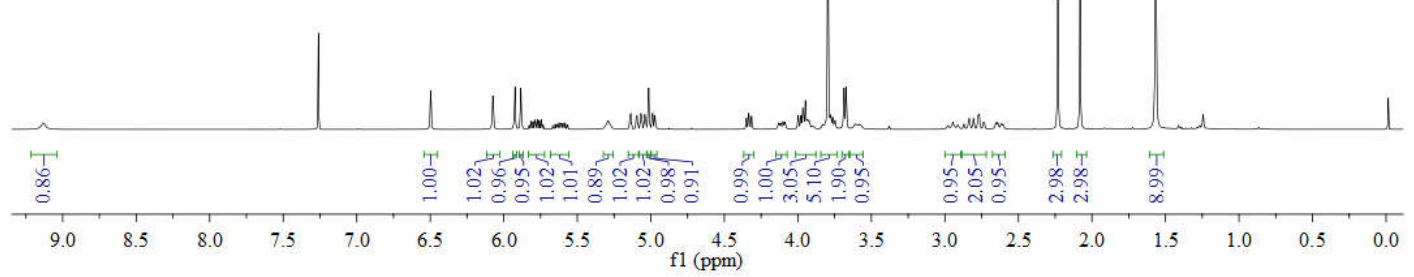


${ }^{1} \mathrm{H}$ NMR spectrum of compound $47\left(400 \mathrm{MHz}, \mathrm{CDCl}_{3}\right)$

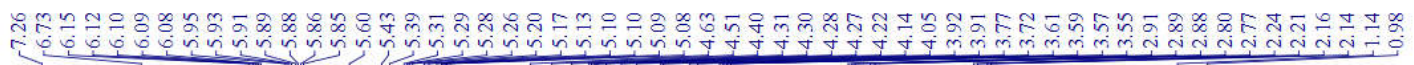

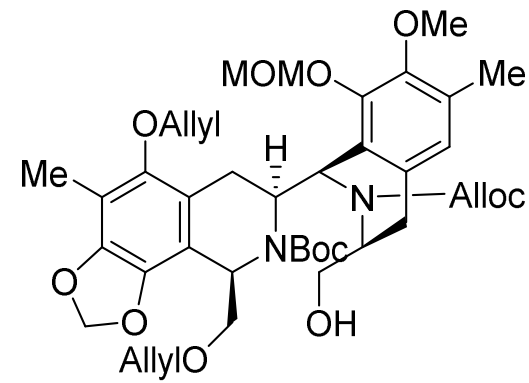

47

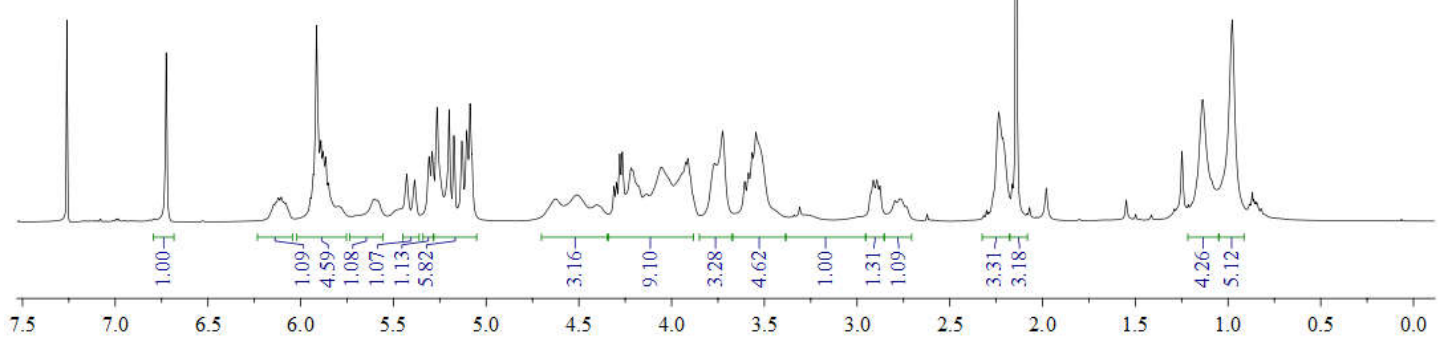

${ }^{13} \mathrm{C}$ NMR spectrum of compound $47\left(100 \mathrm{MHz}, \mathrm{CDCl}_{3}\right)$

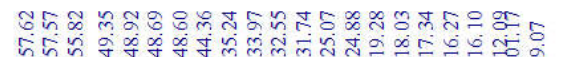

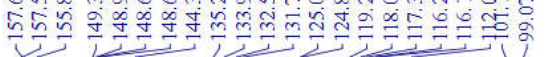

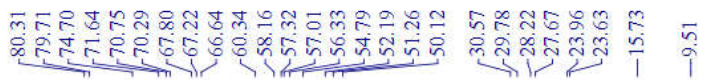

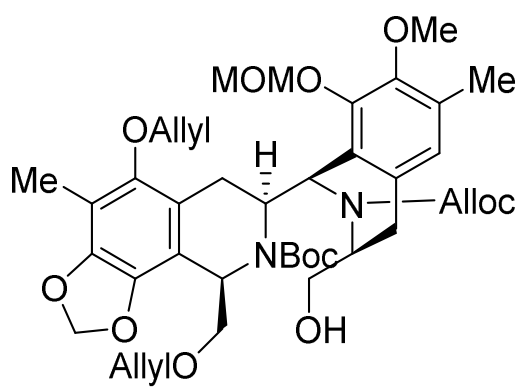

47

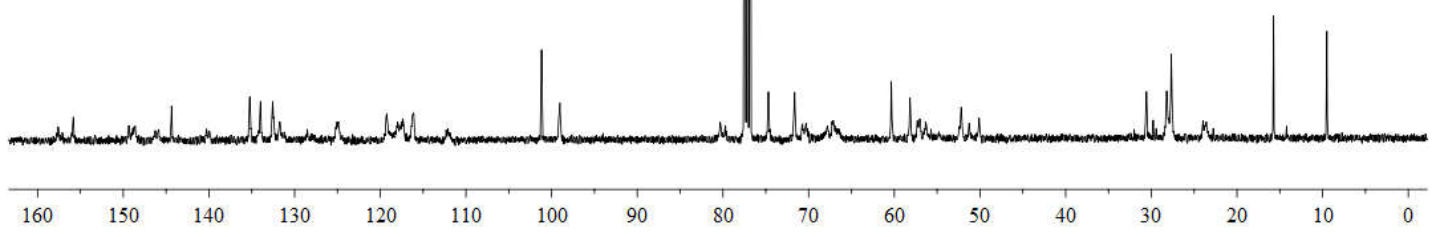


${ }^{1} \mathrm{H}$ NMR spectrum of compound $48\left(400 \mathrm{MHz}, \mathrm{CDCl}_{3}\right)$

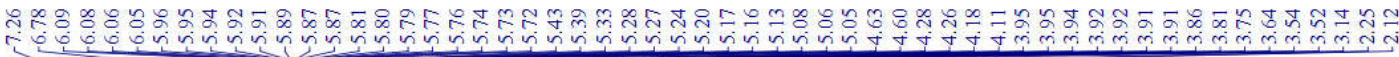

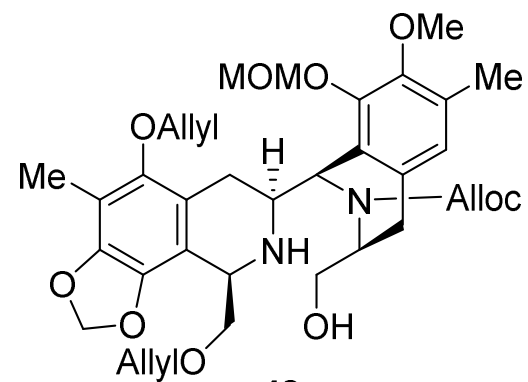

48

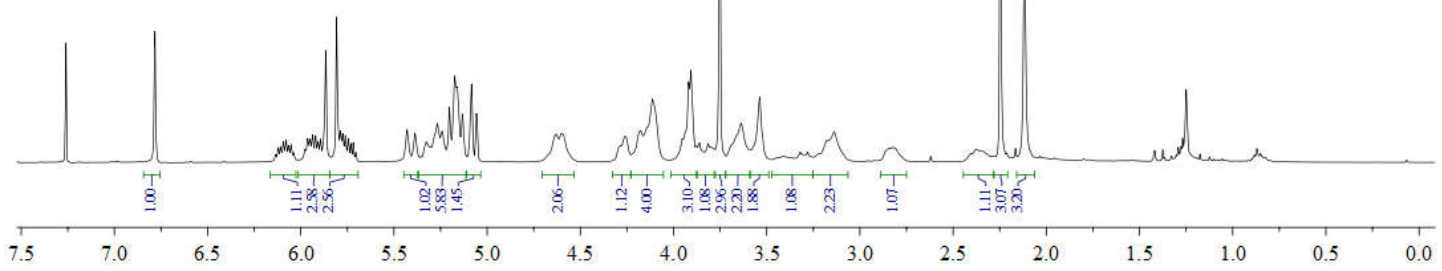

${ }^{13} \mathrm{C}$ NMR spectrum of compound $48\left(100 \mathrm{MHz}, \mathrm{CDCl}_{3}\right)$

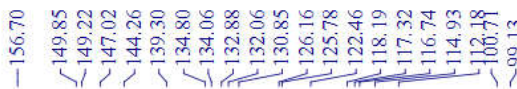

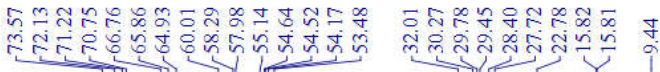

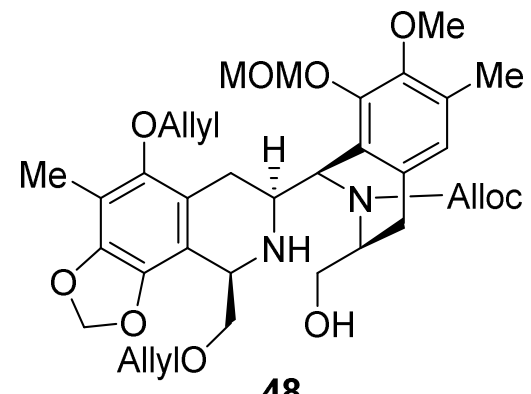
48

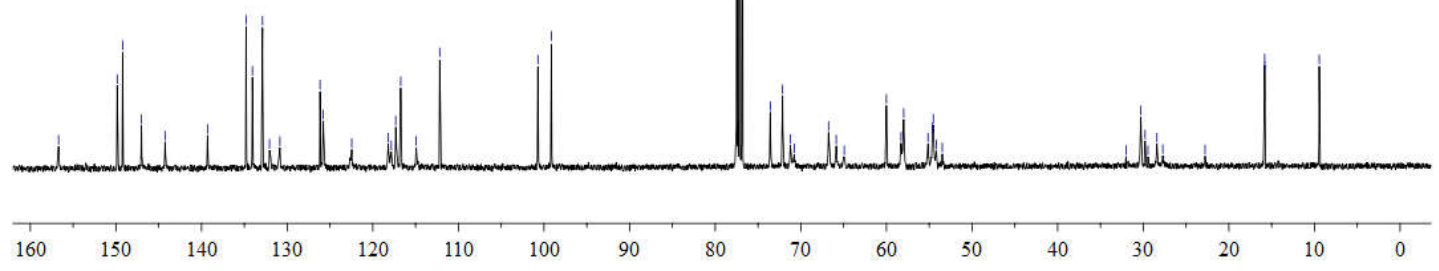


${ }^{1} \mathrm{H}$ NMR spectrum of compound $49\left(400 \mathrm{MHz}, \mathrm{CDCl}_{3}\right)$

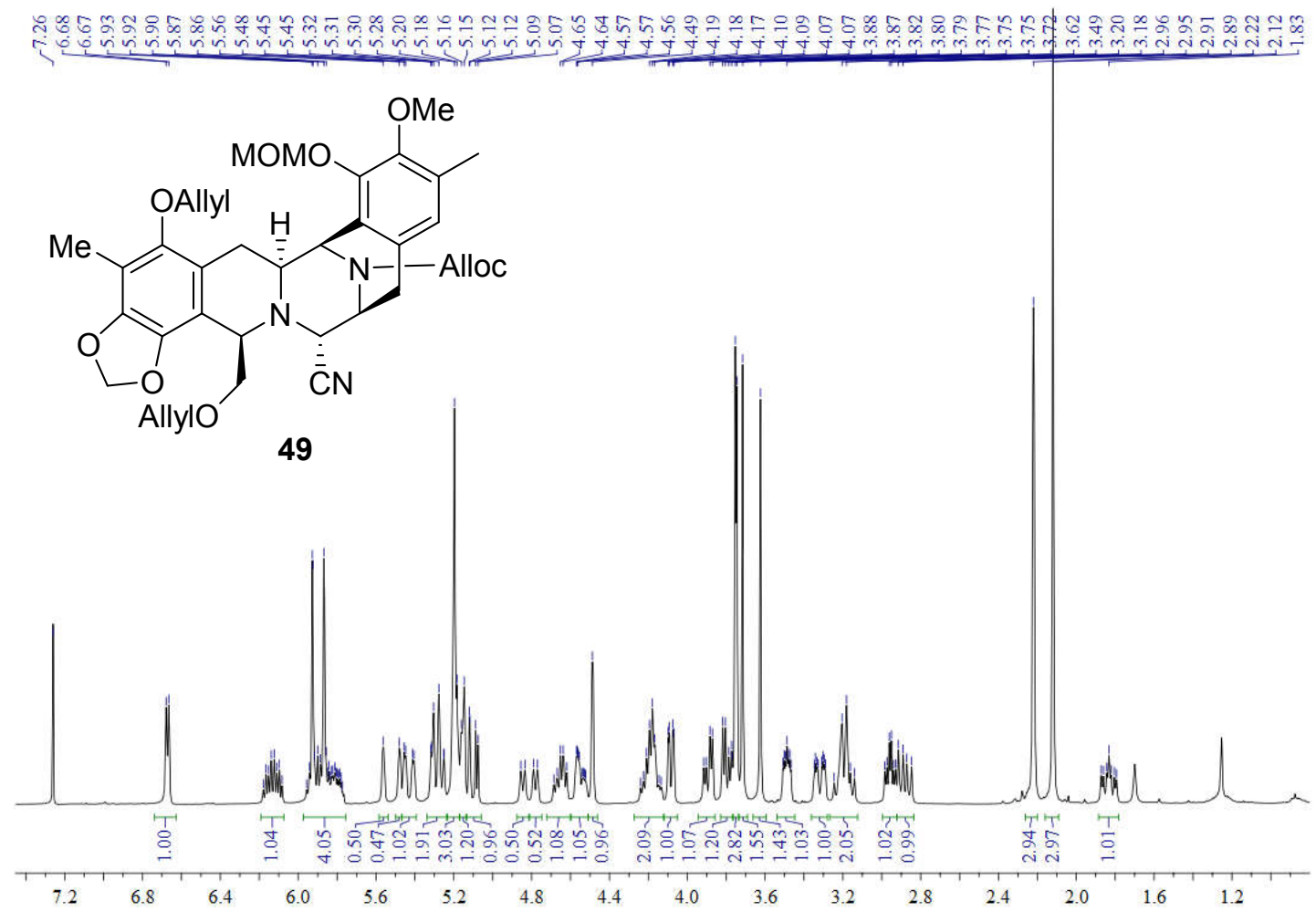

${ }^{13} \mathrm{C}$ NMR spectrum of compound $49\left(100 \mathrm{MHz} \mathrm{CDCl}_{3}\right)$

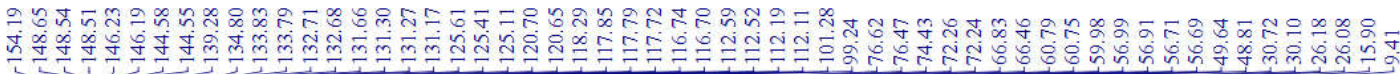

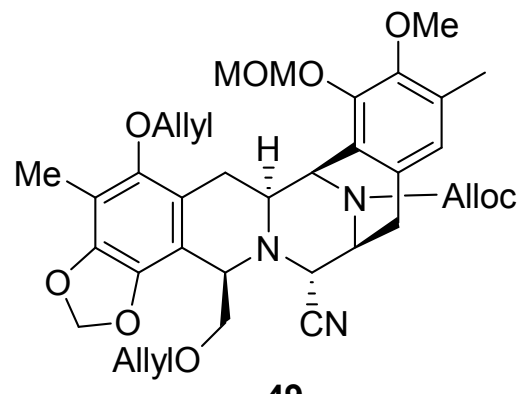

49

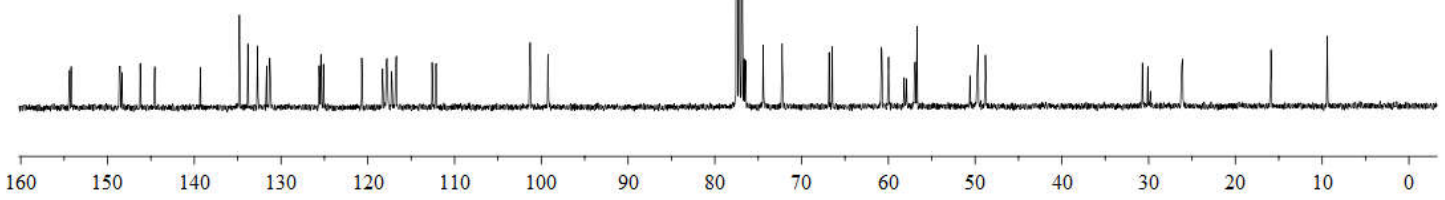

